\title{
Tissue-specific control of midbody microtubule stability by Citron kinase through modulation of TUBB3 phosphorylation
}

\author{
F Sgrò ${ }^{1}$, FT Bianchi ${ }^{1}$, M Falcone ${ }^{1}$, G Pallavicini ${ }^{1}$, M Gai ${ }^{1}$, AMA Chiotto ${ }^{1}$, GE Berto ${ }^{1}$, E Turco ${ }^{1}$, YJ Chang ${ }^{2}$, WB Huttner ${ }^{2}$ and F Di Cunto ${ }^{\star, 1,3}$
}

Cytokinesis, the physical separation of daughter cells at the end of cell cycle, is commonly considered a highly stereotyped phenomenon. However, in some specialized cells this process may involve specific molecular events that are still largely unknown. In mammals, loss of Citron-kinase (CIT-K) leads to massive cytokinesis failure and apoptosis only in neuronal progenitors and in male germ cells, resulting in severe microcephaly and testicular hypoplasia, but the reasons for this specificity are unknown. In this report we show that CIT-K modulates the stability of midbody microtubules and that the expression of tubulin $\beta$-III (TUBB3) is crucial for this phenotype. We observed that TUBB3 is expressed in proliferating CNS progenitors, with a pattern correlating with the susceptibility to CIT-K loss. More importantly, depletion of TUBB3 in CIT-K-dependent cells makes them resistant to CIT-K loss, whereas TUBB3 overexpression increases their sensitivity to CIT-K knockdown. The loss of CIT-K leads to a strong decrease in the phosphorylation of S444 on TUBB3, a post-translational modification associated with microtubule stabilization. CIT-K may promote this event by interacting with TUBB3 and by recruiting at the midbody casein kinase-2 $\alpha$ (CK2 $\alpha)$ that has previously been reported to phosphorylate the S444 residue. Indeed, CK2 $\alpha$ is lost from the midbody in CIT-K-depleted cells. Moreover, expression of the nonphosphorylatable TUBB3 mutant S444A induces cytokinesis failure, whereas expression of the phospho-mimetic mutant S444D rescues the cytokinesis failure induced by both CIT-K and CK2 $\alpha$ loss. Altogether, our findings reveal that expression of relatively low levels of TUBB 3 in mitotic cells can be detrimental for their cytokinesis and underscore the importance of CIT-K in counteracting this event.

Cell Death and Differentiation (2016) 23, 801-813; doi:10.1038/cdd.2015.142; published online 20 November 2015

At the end of the cell division cycle, the daughter cells are physically separated by cytokinesis, a complex process based on dramatic rearrangements of the cytoskeleton and of the membrane trafficking machinery. ${ }^{1-4}$ Because of the highly stereotyped sequence of events that characterize this process and of the strong phylogenetic conservation of the underlying molecular machinery, ${ }^{2,5}$ cytokinesis is currently considered a 'default' biological process, occurring similarly in the different cell types. However, it is well known that specialized proliferating cells are characterized by significant variations of the standard scheme, such as incomplete cytokinesis in trophoblast cells, hepatocytes, Purkinije neurons, cardiomyocytes ${ }^{6}$ and spermatogonia ${ }^{7}$ and asymmetric cytokinesis in meiotic oocytes and in cortical neuroepithelial precursors. ${ }^{8,9}$ Much is known on the molecular machinery responsible for the execution of the 'core' cytokinesis program, with particular regard to the crucial role of Rho small GTPase and of the molecules that locally modulate and/or mediate its activity at the cleavage furrow and at the midbody. ${ }^{10-12}$ Much less is known on the molecules that may regulate cell type-specific aspects of cytokinesis. Citron kinase (CIT-K), a conserved ser/thr protein kinase that binds to active RhoA, ${ }^{13,14}$ is localized at the cleavage furrow and at the midbody of dividing cells. ${ }^{13} \mathrm{CIT}-\mathrm{K}$ was first considered a 'core' cytokinesis protein because it is ubiquitously expressed in proliferating cells, ${ }^{13,15}$ is conserved from insects to mammals ${ }^{16-19}$ and is required by many cultured cell types to complete cytokinesis. ${ }^{15}$ However, the characterization of CIT-K knockout mice and the finding of a spontaneous rat mutant have demonstrated that, in vivo and in mammals, CIT-K is not ubiquitously required. ${ }^{20,21}$ Indeed, CIT-K knockout mice and rats display cytokinesis failure only in few cell types, such as neuronal progenitors ${ }^{21}$ and testicular germ cells. ${ }^{22}$ These cells become polyploid and undergo massive apoptosis in CIT-K - / - animals, leading to a malformative syndrome characterized by severe microcephaly and testicular hypoplasia, associated with ataxia and drug-resistant epilepsy, resulting in death during the first three postnatal weeks. ${ }^{20,21}$ Why the requirement of CIT-K is context specific in mammalian cells is presently unknown. At the molecular level, the function of CIT-K has been principally related to RhoA-dependent actin rearrangements. Indeed, CIT-K can stimulate actin polymerization ${ }^{14,23}$ and has been shown to regulate abscission by stabilizing at the midbody the active form of RhoA and the actin-binding protein Anillin. ${ }^{23,24}$ However, recent results have indicated that CIT-K is also capable of binding microtubules and of promoting midbody maturation by affecting the localization of the kinesins MKLP1 and KIF14 and of the microtubule-bundling protein PRC1. ${ }^{25}$ These results raised the possibility that the function of CIT-K

\footnotetext{
${ }^{1}$ Department of Molecular Biotechnology and Health Sciences, University of Turin, Turin, Italy; ${ }^{2}$ Max-Planck Institute of Molecular Cell Biology and Genetics, Dresden, Germany and ${ }^{3}$ Neuroscience Institute of Turin, Turin, Italy

${ }^{*}$ Corresponding author: F Di Cunto, Department of Molecular Biotechnology and Health Sciences, University of Turin, Via Nizza 52, Turin 10126, Italy. Tel: +39 011 6706409; Fax: +39011 6706432; E-mail: ferdinando.dicunto@unito.it

Abbreviations: CIT-K, citron-kinase; CK2 $\alpha$, casein kinase-2 $\alpha$; FRAP, fluorescence recovery after photobleaching; DP, differentiating progenitor; PP, proliferating progenitor; GRK2, G protein-coupled receptor kinase 2

Received 27.2.15; revised 13.9.15; accepted 29.9.15; Edited by H Ichijo; published online 20.11.15
} 
may also be related to microtubule organization and that context-dependent differences in microtubule stability may condition the requirement for CIT-K during cytokinesis. In this report we show that this is indeed the case. We found that loss of CIT-K leads to destabilization of midbody microtubules and that the sensitivity of dividing mammalian cells to CIT-K inactivation can be modulated by microtubule-affecting drugs. Moreover, the sensitivity of cytokinesis to CIT-K loss, in vitro and in vivo, correlates with the expression of TUBB3, a $\beta$-tubulin isoform expressed in the CIT-K-dependent tissues and in cultured cells that are sensitive to CIT-K inactivation. Finally, we provide evidence that CIT-K can mediate microtubule stabilization by increasing the phosphorylation of TUBB3 on S444, most likely through an indirect mechanism involving its interaction with casein kinase-2a (CK2a).

\section{Results}

During CNS development, the absence of CIT-K affects neurogenic cytokinesis more than proliferating progenitor cytokinesis. A fundamental aspect to unravel the molecular basis of the sensitivity to CIT-K loss is to define which cell divisions are more strictly dependent on CIT-K. The previous finding of many binucleated neurons in the cortex of knockout animals ${ }^{26}$ indicated that neurogenic divisions are specifically affected by CIT-K loss, if compared to gliogenic divisions. However, these studies did not establish whether neurogenic progenitors at different maturation stages are homogeneously or differentially sensitive to CIT-K inactivation. Since in developing CIT-K knockout brains cytokinesis failure is prominently followed by programmed cell death, ${ }^{21}$ to address this issue we analyzed the spatial and temporal distribution of apoptotic cells during neocortical development. In agreement with previous results, apoptotic cells are very rare in the cortical plate and very abundant in the regions populated by early postmitotic neurons, such as the intermediate zone (Figures $1 \mathrm{a}-\mathrm{c}$ ). In addition, apoptotic cells are also detected in the ventricular and subventricular regions that contain both the apical and the basal proliferating progenitors, and their frequency significantly increases from early to middle neurogenesis (Figure 1a). This suggests that the absence of CIT-K may affect the division of differentiating progenitors (DPs) more strongly than the division of proliferating progenitors (PPs). To test this hypothesis, cortical sections were subjected to TUNEL assay, to reveal apoptotic cells, and were immunostained in parallel with antibodies recognizing Pax6 (Figure 1b), Tbr2 (Figure 1c) and Tubb3 (Figures $1 \mathrm{~b}$ and $\mathrm{c}$ ) that identify apical progenitors, basal progenitors or postmitotic neurons, respectively. ${ }^{27}$ Interestingly, although $\sim 60 \%$ of the apoptotic cells were negative for these markers, double positive cells were clearly detected in all cases (Figures 1d-f). However, the frequency of apoptosis in Tubb3-positive cells was much higher than in Pax6-positive or in Tbr2-positive cells (Figure 1g). Altogether, these data indicate that the absence of CIT-K affects principally cell divisions that would produce differentiating neurons, both in the apical and basal regions of the developing neocortex.
CIT-K regulates abscission by increasing the stability of midbody microtubules. Previous results indicated that the primary function of CIT-K, conserved from insects to mammals, is to promote the stability and the maturation of midbody before abscission. ${ }^{15,23-25}$ To assess whether this function may involve the stabilization of midbody microtubules, we measured the ratio of acetylated versus tyrosinated $a$-tubulin at the midbody of control cells and of cells lacking CIT-K. ${ }^{28}$ Indeed, dynamic microtubules contain a relatively high amount of $a$-tubulin tyrosinated at its carboxyterminus, whereas stable microtubules show a high content of $a$-tubulin acetylated at the $\mathrm{K} 40$ residue. ${ }^{29}$ We studied two cellular models characterized by different sensitivity to CIT-K loss and by different physiological relevance: cultures of proliferating neocortical neuronal progenitors obtained from control or from Cit-K knockout mice at embryonic day (E)12.5 and HeLa cells depleted of CIT-K by RNAi. In both models cytokinesis fails after cells have reached the midbody stage, with a penetrance of $\sim 90 \%$ in the case of neuronal progenitors and of $30 \%$ in the case of HeLa cells (Gai et al. ${ }^{23}$ and data not shown). The analysis revealed that the ratio between tyrosinated and acetylated tubulin at the midbody is increased by approximately fourfold in the Cit-K knockout neuronal progenitors (Figure $2 \mathrm{a}$ ) and by twofold in CIT-Kdepleted HeLa cells (Figure 2b). The same ratio is also increased outside of the midbody in telophase progenitors of Cit-K-/ - mice, although to a lesser extent than at the midbody (Supplementary Figure S1A). In contrast, the ratio is not significantly different from the control in nondividing progenitors and HeLa cells, and outside of the midbody in telophase HeLa cells (Supplementary Figures S1A and B). We then evaluated whether the altered ratio between tyrosinated and acetylated tubulin produced by CIT-K loss could depend on KIF14, a microtubule-binding protein that has been shown to work immediately downstream of CIT-K in Drosophila ${ }^{25}$ and has been proposed to do so also in mammalian cells ${ }^{15,25}$ by depleting this protein in cultured wild-type neuronal progenitors and in HeLa cells (Supplementary Figure S2A). However, cells depleted for KIF14 did not show significant differences when compared with the corresponding controls (Figures $2 a$ and b). This result suggests that CIT-K affects midbody microtubules' stability independently from KIF14, especially in cells showing the highest sensitivity to CIT-K loss. To address more directly this phenotype, we evaluated the turnover rate of $a$-tubulin at the midbody of control cells and of CIT-Kdepleted cells by performing fluorescence recovery after photobleaching (FRAP) ${ }^{30}$ in HeLa cells stably expressing RFP-tagged $a$-tubulin. This test revealed that knockdown of CIT-K reduces the half-time of recovery by $50 \%$ (Figure $2 \mathrm{c}$ ). Finally, we measured the growth rate of midbody microtubule plus ends ${ }^{31}$ by tracking comets of the plus-tip-binding protein EB1 fused to GFP and transiently transfected in control or in CIT-K-depleted HeLa cells. Even in this case a significant difference was observed, with the speed of comets in CIT-Kdepleted cells being more than the double of the speed in the control (Figure 2d and Supplementary Movies S1 and S2). These results show that CIT-K inactivation increases the turnover of midbody microtubule. On this basis, we predicted that altering microtubules dynamics by pharmacological 
a

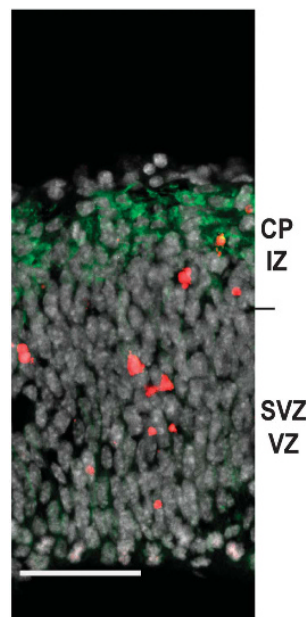

TUN TUJ DAPI
E14.5

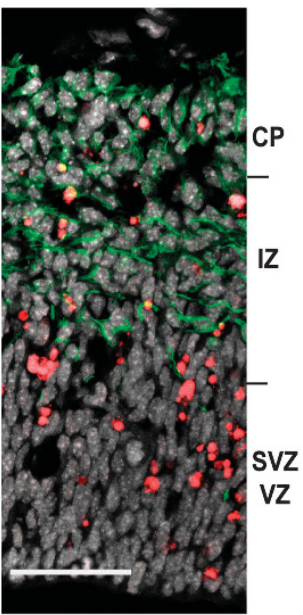

SVZ b

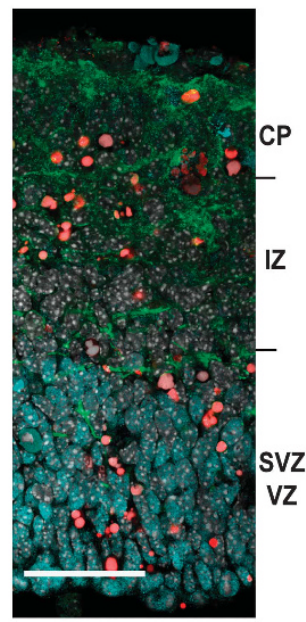

TUN TuJ Pax6 DAPI

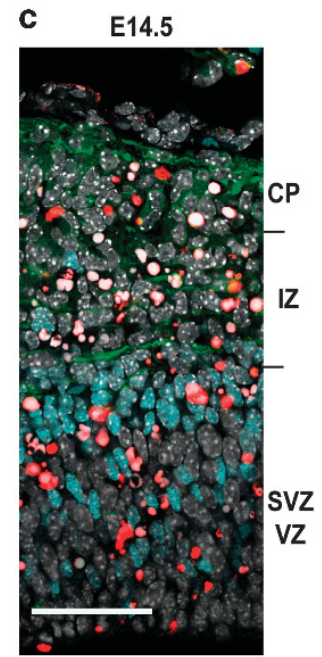

TUN TuJ Tbr2 DAPI

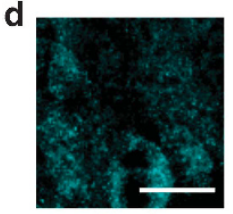

Pax6

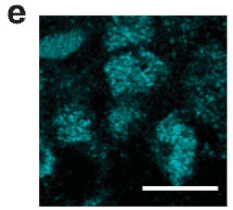

Tbr2

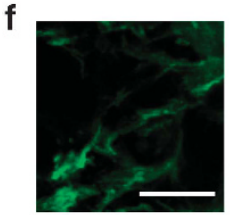

TuJ

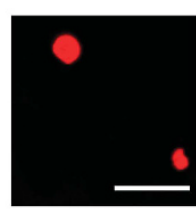

TUN

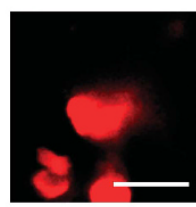

TUN

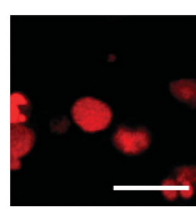

TUN

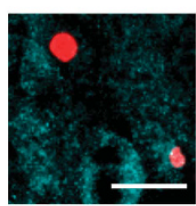

Merge

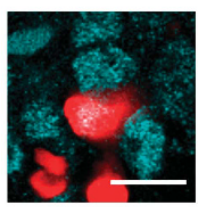

Merge

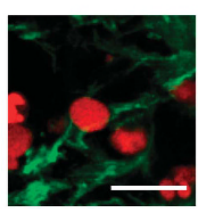

Merge

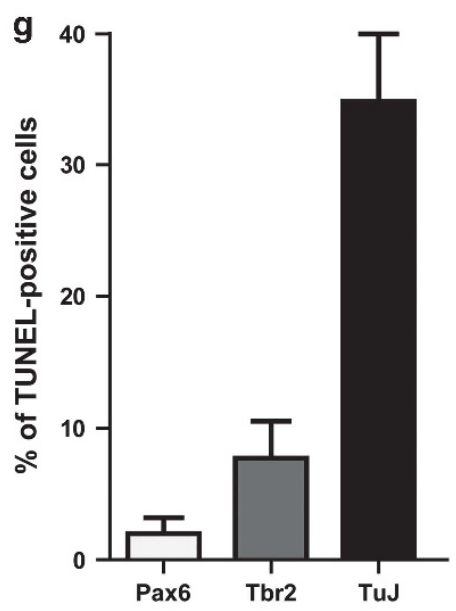

Figure 1 During development of the neocortex, the loss of CIT-K mainly affects neurogenic divisions. (a-c) Low-magnification micrographs of mouse dorsal telencephalon sections obtained from CIT-K knockout embryos at the indicated developmental ages. All the sections were labeled by TUNEL (TUN) to reveal apoptotic cells. Sections were also stained for Tubb3 (TuJ), Pax6 and Tbr2, as indicated, and counterstained for DNA with DAPI. VZ, ventricular zone; SVZ, subventricular zone; IZ, intermediate zone; CP, cortical plate. Scale bars $=50 \mu \mathrm{m}$. (d-f) High-magnification fields of sections shown in $\mathbf{b}(\mathbf{d})$ and $\mathbf{c}(\mathbf{e}$ and $\mathbf{f})$, showing TUNEL-positive cells stained with the indicated markers. Scale bars $=5 \mu \mathrm{m}$. (g) Quantification of cells positive for the indicated markers and for TUNEL detected in sections from E14.5 CIT-K knockout embryos. In this figure and in all the following, bars represent average values of at least three independent animals. Error bars =S.E.M.

treatment should result in different sensitivity of cells to CIT-K inactivation. We therefore assessed whether stabilization or destabilization of microtubules, by paclitaxel or nocodazole, respectively, can modify the frequency of cytokinesis failure in HeLa cells. To avoid the confounding effects that can be produced by the activity of these drugs in the other mitotic phases, control and CIT-K-depleted HeLa cells were synchronized and treated with the drugs during telophase, that is, after the genetic material had already been segregated by spindle microtubules. The drugs were used at a low concentration, which is known to specifically affect dynamic microtubules, ${ }^{32,33}$ and the percentage of binucleated cells was then measured. Treatment with either drugs did not change significantly the rate of cytokinesis failure in control cells (Figure 2e). Interestingly, microtubule destabilization by nocodazole increased the percentage of binucleated cells after CIT-K knockdown (Figure 2e). Moreover, treatment of CIT-K-depleted cells with Paclitaxel reduced the rate of cytokinesis failure almost to control values (Figure 2e). Conversely, we found that nocodazole treatment rescues the cytokinesis delay phenotype induced by CIT-K overexpression that we previously described ${ }^{23}$ (Figure 2f). Altogether, these results indicate that CIT-K regulates abscission by increasing the stability of midbody microtubules and that differential stability of dynamic microtubules during cytokinesis may determine the sensitivity of cells to CIT-K loss. 

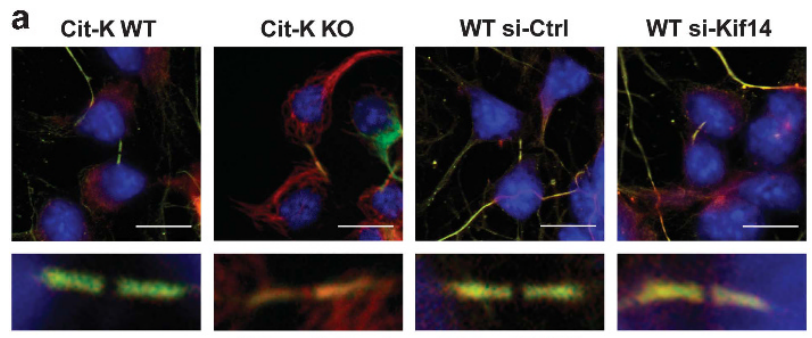

Tyr- $\alpha-$ Tub Ac= $\alpha=$ Tub DAPI

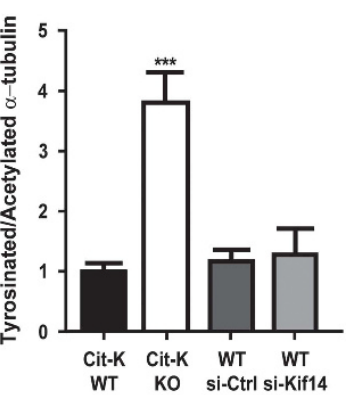

b

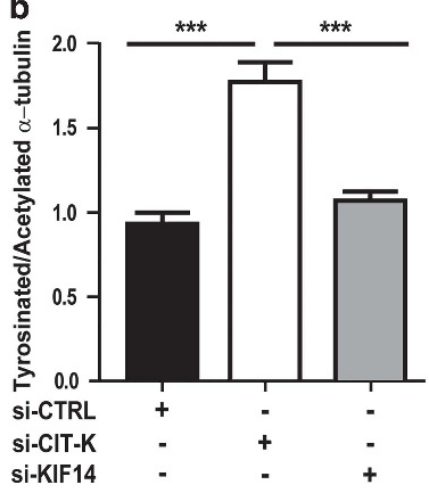

C

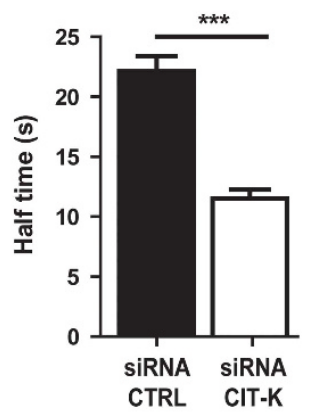

d

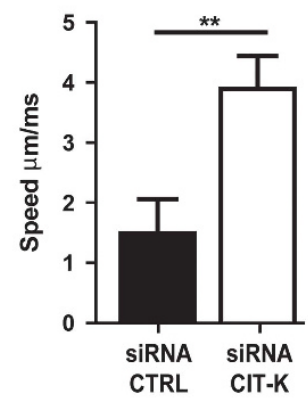

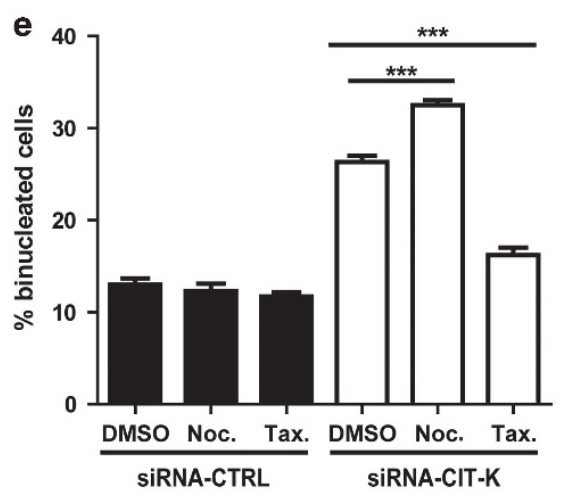

f

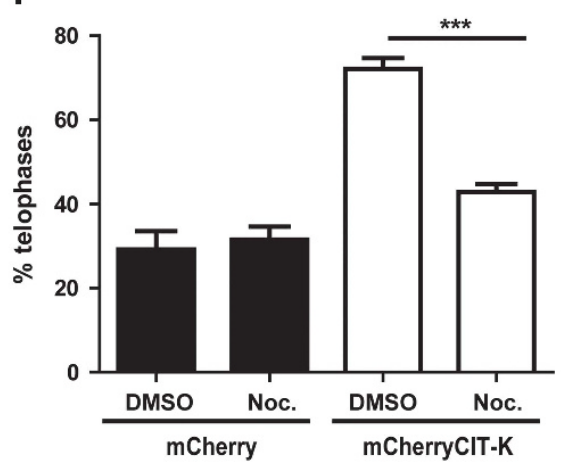

Figure 2 The stability of midbody microtubules decreases after CIT-K loss and modifies the severity of the CIT-K knockdown phenotype. (a) Cultures of dissociated proliferating neuronal progenitors were obtained from the dorsolateral telencephalon of E12.5 CIT-K wild type (Cit-K WT) or knockout (Cit-K KO) embryos. At $48 \mathrm{~h}$ after plating, cells were stained with antibodies anti-tyrosinated (Tyr- $\alpha$-Tub) or anti-acetylated (Ac- $\alpha$-Tub) $\alpha$-tubulin. To evaluate the role of Kif14, WT cells were nucleofected before plating with control (WT si-Ctrl) or with Kif14-specific (WT siRNA) double-stranded RNA oligonucleotides. The ratio between the intensity of Ac- $\alpha$-Tub on the intensity of Tyr- $\alpha$-Tub was then measured at single midbodies of late telophase cells $(n=30)$ by confocal microscopy. DNA was stained by DAPI. Scale bars $=10 \mu \mathrm{m}$. (b) The same analysis performed in (a) was repeated on HeLa cells treated for $48 \mathrm{~h}$ with either control siRNA or with previously validated siRNAs designed on CIT-K mRNA ${ }^{23}$ or KIF14. ${ }^{15}$ In the case of CIT-K, similar results were obtained using previously validated shRNA-expressing plasmids ${ }^{23}$ (data not shown). (c) HeLa cells stabling expressing RFP- $\alpha$-tubulin were treated as in (b). After $48 \mathrm{~h}$, the fluorescence of midbodies ( $n=20$ per each condition) was bleached by a $568 \mathrm{~nm}$ laser pulse. Recovery kinetics were measured in midbodies of constant size and, after background subtraction, were normalized to pre- and last post-bleach frame. (d) HeLa cells transiently transfected with fluorescent +tip protein eGFP-EB1 were treated $24 \mathrm{~h}$ after transfection as in (b). After additional $48 \mathrm{~h}$, the speed of eGFP-EB1 comets was measured by time-lapse confocal microscopy in a $20 \mu \mathrm{m}$-wide region of interest (ROI) centered on late telophases of cells expressing intermediate levels of eGFP-EB1 using $63 \times 1.3$ objective at 1 frame/500 ms for $2 \mathrm{mi}$ ( $n=25$ for each condition). (e) HeLa cells were transfected with control or ClT-K siRNAs and synchronized with the thymidine/nocodazole method. At $180 \mathrm{~min}$ after complete nocodazole washout, nocodazole (Noc., $50 \mathrm{nM}$ ), paclitaxel (Tax., $1 \mathrm{nM}$ ) or diluent (DMSO) were added for $120 \mathrm{~min}$. Cells were then fixed and stained with anti- $\alpha$-tubulin antibodies and DAPI and the percentage of binucleated cells was determined. (f) HeLa cells were transfected with a control (mCherry) or with mCherry-CIT-K expression constructs and synchronized as in (e). At 180 min after complete nocodazole washout, nocodazole (Noc., $50 \mathrm{nM}$ ) or DMSO were added for $120 \mathrm{~min}$ in HeLa cells. The percentage of cells in telophase was determined after processing the samples as in (e). Error bars $=$ S.E.M. Statistical significance of differences in average was established using Student's two-tailed $t$-test. ${ }^{\star \star} P<0.01 ;{ }^{\star \star \star} P<0.001$

Tubb3 is expressed in proliferating cortical progenitors and modifies the sensitivity of HeLa cells to CIT-K loss. Based on the above data, we hypothesized that the strong sensitivity of neurogenic divisions to CIT-K inactivation may depend on intrinsic alterations of midbody microtubule stability, caused by the gene expression changes occurring in neuronal precursors upon commitment to terminal differentiation. We therefore asked which genes functionally related to the microtubule cytoskeleton may undergo the strongest expression changes in this particular developmental window. To address this question, we took advantage of a recent study $^{34}$ in which the transcriptome of neocortical PPs, DPs 
and neurons was carefully characterized by RNA sequencing. Starting from this data set, we ranked the genes associated with the gene ontology (GO) keyword 'microtubule' for the $P$-value of the expression difference measured between PPs and DPs (Supplementary Table S1). Among the genes showing the strongest difference, we found the $\beta$-tubulin subunit Tubb3 whose mRNA is approximately sixfold more abundant in DP than in PP (Supplementary Table S1). This result was particularly interesting in light of previous studies showing that, in human tumor cells, TUBB3-incorporating microtubules have increased dynamic instability. ${ }^{35,36}$ However, although the expression of Tubb3 has been described in some proliferating neuronal precursors of the telencephalon ${ }^{37,38}$ and of the spinal cord, ${ }^{39}$ this finding would be in apparent contrast with the strongly consolidated view that Tubb3 is a specific marker of postmitotic neurons. Therefore, we asked whether neuronal progenitors could express detectable amounts of Tubb3 protein during mitosis and whether this expression may correlate with neurogenesis. We first analyzed E12.5 progenitor cultures in which we observed that the anti-Tubb3 antibody TuJ stains mitotic spindles in a high percentage of cells, even when they still express stem cell markers such as nestin (Figure 3a). We detected clear TuJ positivity in all microtubule-based mitotic structures, such as spindle microtubules, central spindle and midbody microtubules (Figure 3b). A similar pattern was observed even in prometaphases and metaphases of neuronal progenitors obtained from Cit-K-/- mice (Figure $3 \mathrm{~b}$ ). However, in the telophases observed in Cit-K-/- cultures, the TuJ signal appears less defined than in controls, with a reduced number of filaments and an increase in dot-like structures (Figure 3b). In the in vivo situation, TuJ-positive prometaphases and metaphases can also be detected in the proliferating neocortex after E12.5, especially at the border between the SVZ and the $I Z$, but a careful quantification of the ratio between Tubb3positive and -negative mitoses is hampered by the high expression of Tubb3 in the neighboring differentiating neurons (data not shown). For this reason, to evaluate the correlation between mitotic expression of Tubb3 and neurogenesis, we analyzed the midbodies of apically dividing cells that show a high signal-to-noise ratio because of the low expression of Tubb3 in the VZ and of the high concentration of microtubules in the midbody. Interestingly, we found that the Tubb3-positive midbodies are $\sim 5 \%$ at E12.5 and $>30 \%$ at E14.5 (Figures 3c and d), an increase well correlated with the increase of neurogenic divisions that takes place between the two stages. ${ }^{40}$ The above data show that the expression of Tubb3 in proliferating cortical progenitors, at levels lower than in differentiating neurons but still clearly detectable, is much more widespread than previously believed and suggest that the incorporation of Tubb3 in midbody microtubules could be one of the factors that render neurogenic divisions sensitive to CIT$\mathrm{K}$ inactivation. To functionally test this hypothesis we resorted to HeLa cells in which we increased (Supplementary Figures S2B and C) or decreased (Supplementary Figure S2D) the levels of TUBB3, concomitantly with CIT-K knockdown. In overexpressing cells, the levels of exogenous TUBB3 was comparable to the level of the endogenous protein (Supplementary Figure S2B), if considering that the transfection efficiency was approximately equal to $30 \%$. Moreover, the exogenous protein appears to be incorporated in microtubules, including those of the midbody (Supplementary Figure S2C), as previously reported. ${ }^{41}$ The overexpression (Figure $4 \mathrm{a}$ ) or knockdown (Figure 4b) of TUBB3 had no effect on the basal rate of cytokinesis failure. The overexpression of TUBB3 in CITK-depleted HeLa cells significantly increased the ratio of binucleated cells generated within the observation time (Figure 4a). Conversely, the rate of cytokinesis failure induced by CIT-K RNAi was significantly reduced in cells co-depleted for TUBB3 (Figure 4b). In contrast, no significant changes were detected when CIT-K-depleted HeLa cells were co-depleted for the tubulin $\beta$-subunit TUBB2A (Supplementary Figure S2E and Figure $4 b$ ). The knockdown of TUBB3, but not of TUBB2A, also reverted the increased ratio between tyrosinated and acetylated $a$-tubulin at the midbody (Figure 4c). Neither CIT-K nor TUBB3 knockdown significantly altered the ratio between tyrosinated and acetylated $a$-tubulin in the cytoplasm of telophase cells outside of the midbody or in the cytoplasm of cells not in mitosis (Supplementary Figure S1B). Finally, the knockdown of TUBB3 reverted the increase in the turnover of $a$-tubulin at the midbody of CIT-K-depleted cells, whereas the knockdown of TUBB2A had no significant effects (Figure 4d). These results indicate that the expression of TUBB3 specifically modifies the phenotype induced by CIT-K inactivation, both in terms of midbody microtubule dynamics and of cytokinesis failure.

CIT-K affects TUBB3 phosphorylation: role of S444.

We next asked how CIT-K may counteract the effects of TUBB3 expression on microtubule dynamics. To address this issue we performed different types of biochemical analysis on postnatal day 4 (P4) cerebellum from control and Cit-K knockout mice. We chose this tissue because the large majority of cells that compose it are cerebellar granules that are dramatically affected by the loss of CIT-K. ${ }^{21}$ Moreover, at $\mathrm{P} 4$, cerebellar granules' progenitors are intensely proliferating and the cellular composition of the control and of the knockout tissues are much more similar than at later stages, allowing to better highlight the primary defects with respect to the secondary events related to the different ratio between the proliferating and differentiated populations. ${ }^{21}$ Western blot analysis revealed that the loss of Cit- $\mathrm{K}$ does not affect Tubb3 total levels (Figures $5 \mathrm{a}$ and b). Interestingly, reciprocal co-immunoprecipitation assays showed that Cit-K and Tubb3 are in a same physical complex in vivo (Figures $5 a$ and b). Notably, Tubb3 was not found in the Citron immunoprecipitate obtained from Cit-K knockout cerebellum (Figure 5b), not only confirming the specificity of the reagents, but also indicating that the complex must be specific for Cit-K and for proliferating granule precursors. Indeed, Cit-K knockout cerebella express normal levels of CIT-N, a kinase-lacking isoform of CIT-K produced in postmitotic neural cells. ${ }^{42} \mathrm{We}$ then asked whether CIT-K affects TUBB3 post-translational modifications. It has previously been reported that TUBB3 is the main $\beta$-tubulin isoform phosphorylated in brain, and that a prominent phosphorylation site is $S 444 .{ }^{36,43}$ Moreover, it has been shown that dephosphorylated TUBB3 inhibits microtubule assembly. ${ }^{36}$ Therefore, we analyzed whether loss of CIT-K may significantly affect Tubb3 phosphorylation, with particular regard to the $\mathrm{S} 444$ residue. We separated by 
a

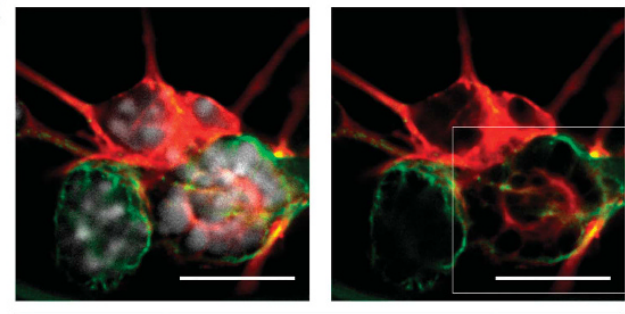

TuJ Nestin DAPI

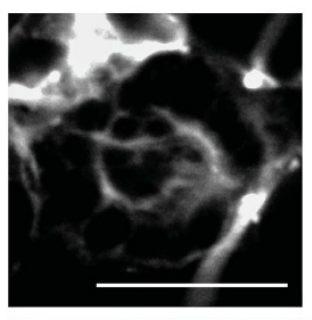

TuJ

b

Cit $\mathrm{K}+/+$

Cit K - -
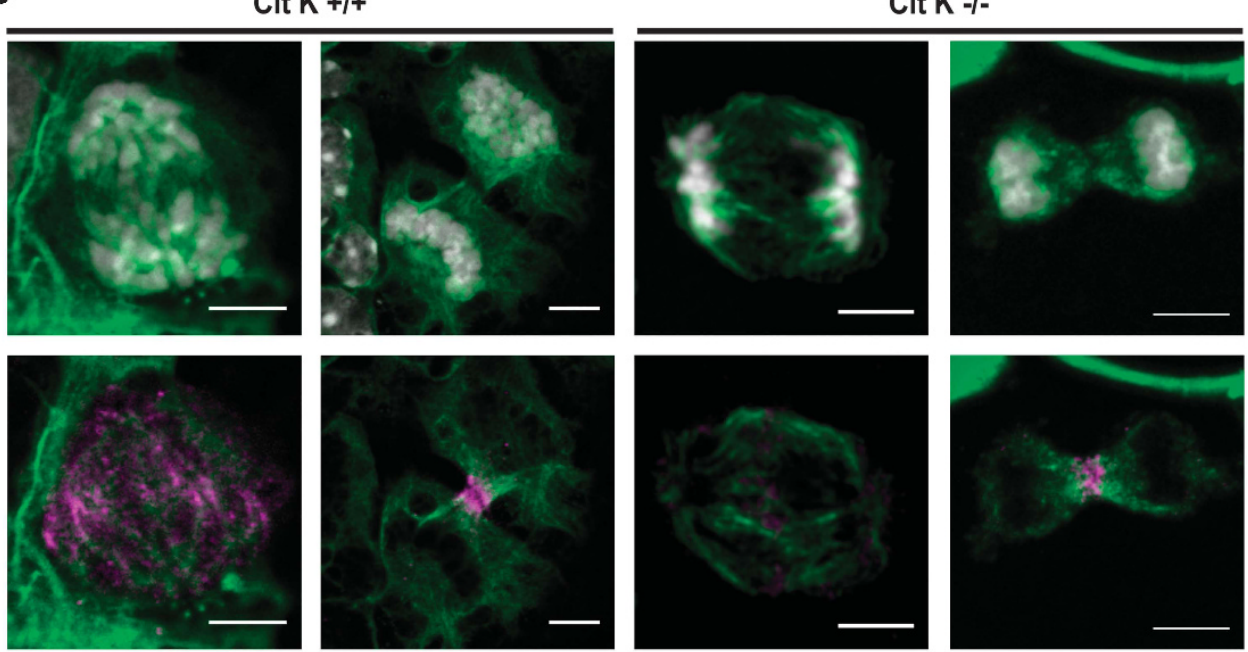

TuJ Aurora B DAPI
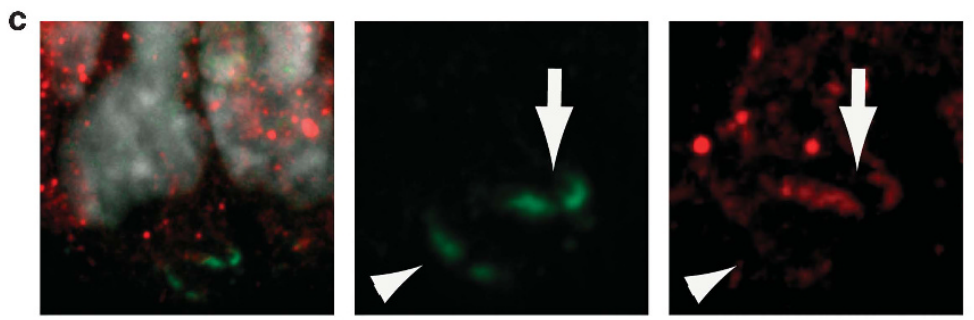

TuJ AuroraB DAPI

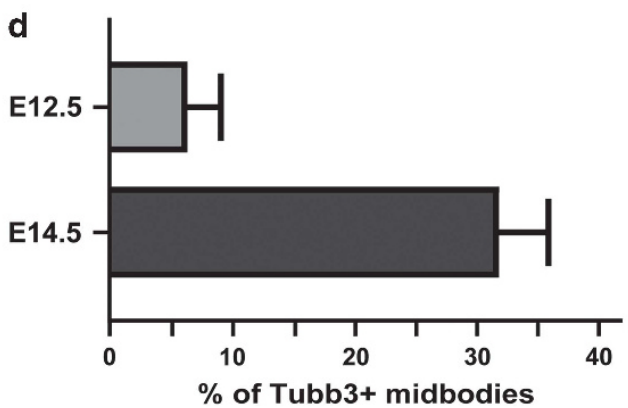

Figure 3 Expression of Tubb3 in proliferating neuronal progenitors. (a) Cultures of proliferating neuronal progenitors were prepared from the dorsolateral telencephalon of E12.5 wild-type mice and processed for IF with the indicated markers. A high-magnification field is shown containing a neuron, strongly positive for TuJ, a nestin-positive progenitor negative for TuJ and a mitotic nestin-positive progenitor showing a TuJ-positive spindle (also shown in magnification in the TuJ channel). Scale bar $=5 \mu \mathrm{m}$. (b) Tubb3 (TuJ)-positive anaphase (left panel) and telophase (right panel), respectively, obtained from Cit-K+/+or $-1-$ mice counterstained for DNA (upper panels) and for the mitotic protein Aurora B (lower panels). Scale bar $=2.5 \mu \mathrm{m}$. (c) Two midbodies located in the apical region of a E14.5 wild-type embryo cortex, identified by means of their positivity for Aurora B, showing a Tubb3 signal lower (arrowhead) or higher (arrow) than the chosen threshold. Scale bar $=2.5 \mu \mathrm{m}$. (d) Quantification of Tubb3-positive midbodies determined as in (c) at the indicated ages. Error bars = S.E.M 
a
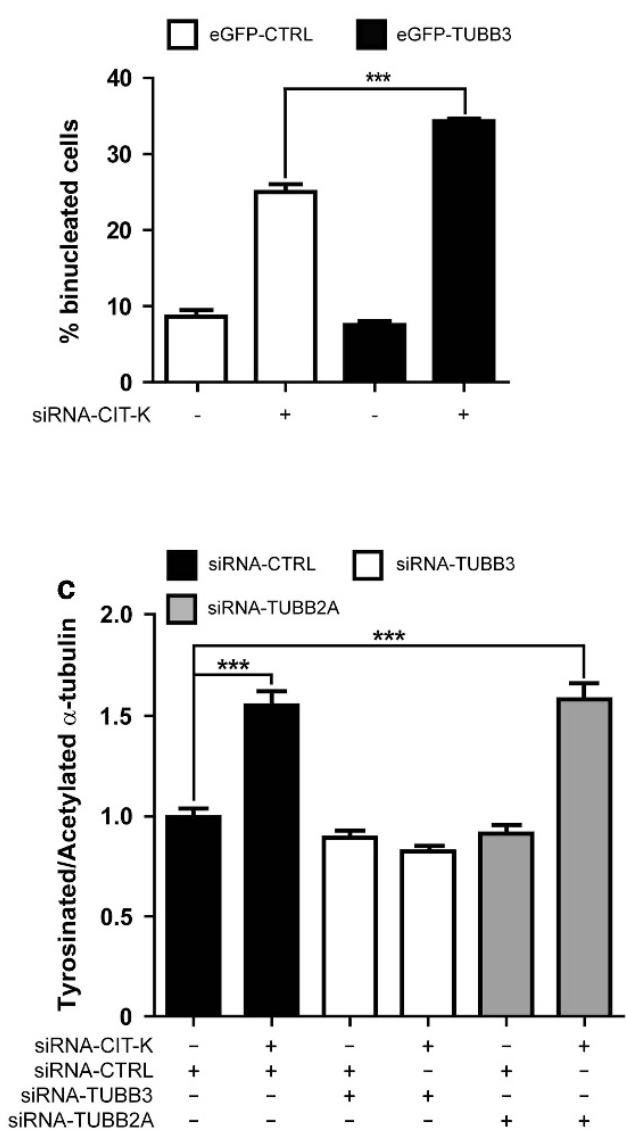
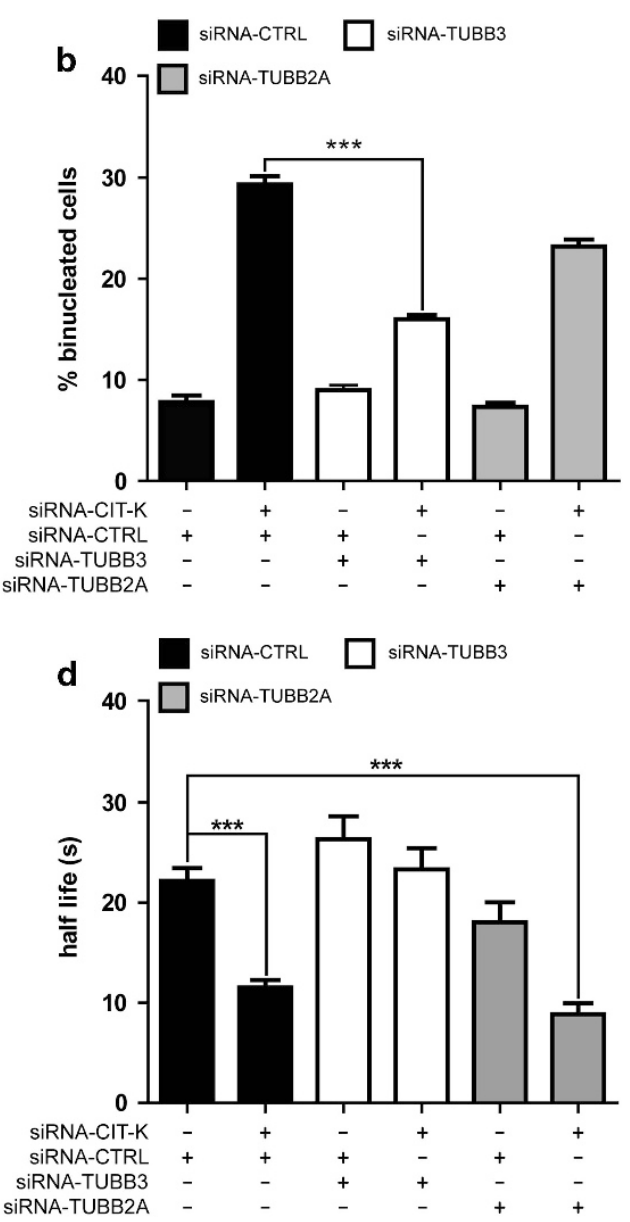

Figure 4 Effects of TUBB3 levels on the sensitivity of HeLa cells to CIT-K depletion. (a) HeLa cells were transiently transfected with eGFP-TUBB3 and after $24 \mathrm{~h}$ were transfected with control (CTRL) or with CIT-K-specific siRNAs. After $48 \mathrm{~h}$, cells were stained with anti- $\alpha$-tubulin antibodies and DAPI and the percentage of binucleated cells was quantified. (b) HeLa cells were transfected with the indicated combinations of siRNAs. After $48 \mathrm{~h}$, the percentage of binucleated cells was quantified as in (a). (c) HeLa cells were transfected with the indicated combinations of siRNAs. After $48 \mathrm{~h}$, the ratio between tyrosinated and acetylated $\alpha$-tubulin was quantified as in Figure 1. (d) HeLa cells stabling expressing RFP- $\alpha$-tubulin were transfected with the indicated combinations of siRNAs. FRAP analysis was then performed as described in Figure 1c. Error bars $=$ S.E.M. Statistical significance of differences in average was established using Student's two-tailed $t$-test. ${ }^{* * *} P<0.001$. The knockdown of the targeted proteins and the expression of the recombinant proteins were confirmed by western blotting (Supplementary Figure S2)

2D gel electrophoresis the proteins extracted from wild-type and Cit-K-I- cerebella and revealed Tubb3 by western blotting. This analysis showed in controls a major spot characterized by an isoelectric point ( $p l)$ compatible with the pl expected for Tubb3. In addition, consistent with previous results, ${ }^{44}$ a less abundant but prominent acidic spot was detected (Figure 5c). Strikingly, the second spot was dramatically reduced in Cit-K-l-2D blots (Figure $5 \mathrm{C}$ ). A similar pattern in TUBB3 2D western was observed by analyzing lysates of HeLa cells, although in this case the intensity of the acidic spot was much less than the intensity detected in mouse cerebellum (Supplementary Figure S3). Even in this case, the acidic spot became almost undetectable when CIT-K was depleted by RNAi (Supplementary Figure S3). As it has previously been shown that TUBB3 phosphorylation is resistant to treatment with many phosphatases but not to erythrocyte PP2A, ${ }^{36}$ to address whether the acidic spot corresponds to a phosphorylated form of Tubb3, we pretreated lysates of wild-type cerebella with lambda phosphatase or with PP2A and analyzed them by $2 \mathrm{D}$ western blot. In samples treated with lambda phosphatase the TUBB3-reactive acidic spot did not disappear, but was more clearly resolved as two separate signals (Figure $5 d$ ). In contrast, the acidic spot was completely undetectable after PP2A treatment (Figure $5 \mathrm{~d}$ ). We then asked whether phosphorylation of S444 is a prominent modification of the Tubb3 molecules that migrate into the acidic spot. As there are no available antibodies specifically recognizing this modification, we resorted to an antiserum that does not recognize Tubb3 when it is phosphorylated on $\mathrm{S} 444 .^{45}$ Interestingly, although this antibody very efficiently recognized the principal Tubb3 spot, it gave no detectable signal in correspondence of the acidic spot detected by the TuJ antibody (Figure $5 d$ ). To finally prove that phosphorylation of S444 is mainly responsible for the acidic spot, we expressed in HeLa cells a wild-type GFP-TUBB3 fusion or a nonphosphorylatable mutant form in which serine-444 was replaced by alanine (S444A) and then revealed the recombinant proteins by $2 \mathrm{D}$ western blotting with anti-GFP antibodies. This analysis showed that the wild-type fusion displays a 
a

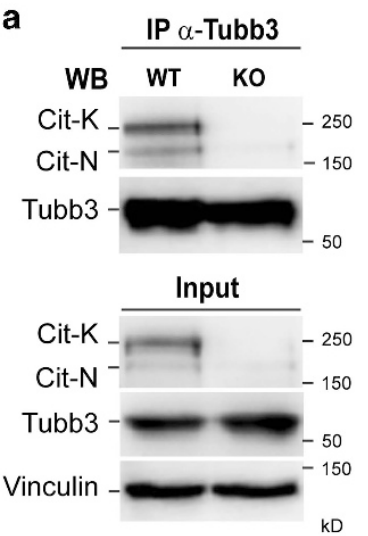

d

Cit-K WT P4 Cerebellum

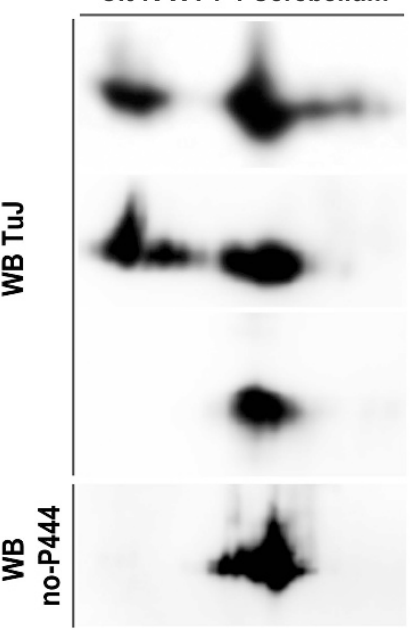

e

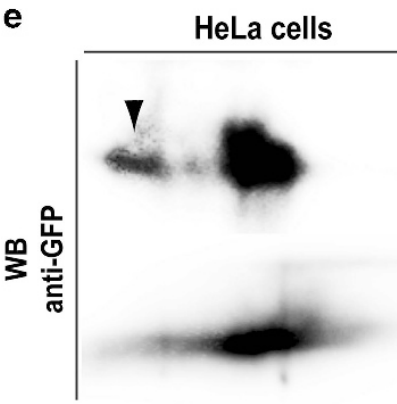

b

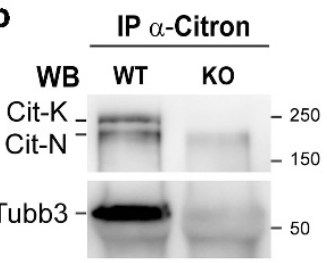

Input

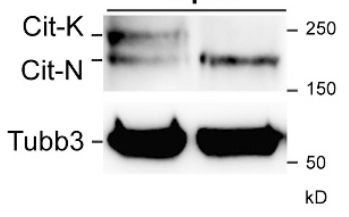

c
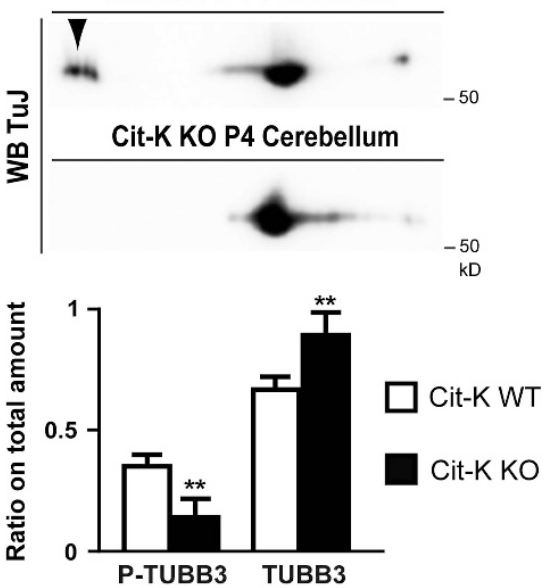

Ctrl

$\mathrm{PP} \lambda$

PP2A

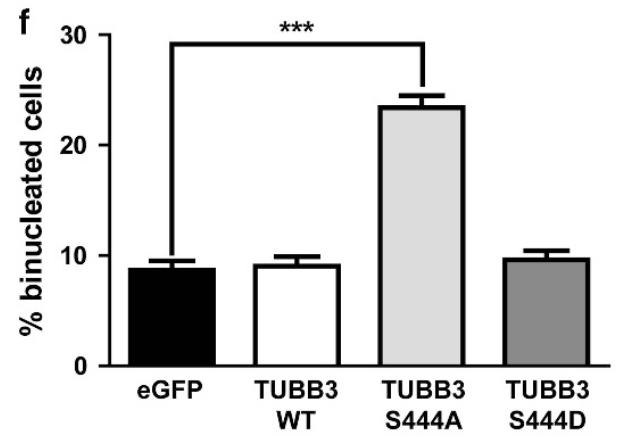

Ctrl

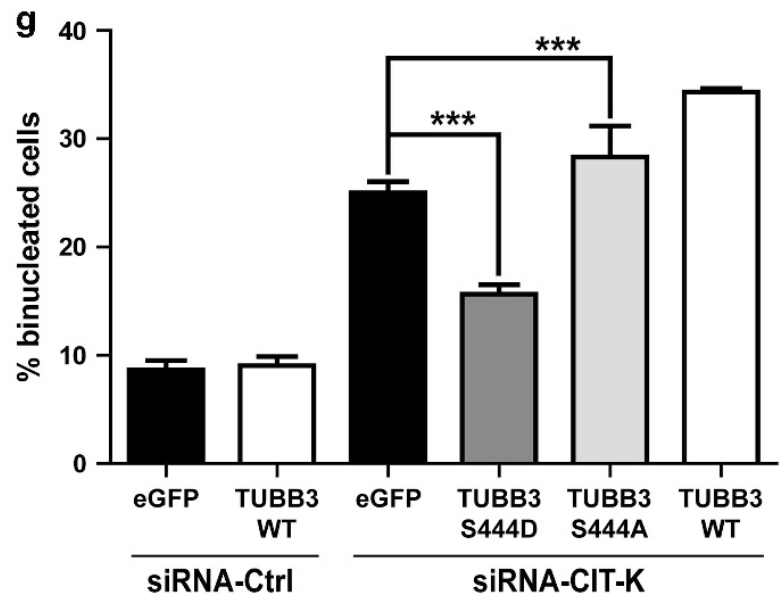

Figure 5 CIT-K physically associates with Tubb3 in proliferating CNS and affects the phosphorylation of its S444 residue. (a and $\mathbf{b}$ ) Total cell lysates from P4 wild-type (WT) and Cit-K knockout (KO) mouse cerebellum were immunoprecipitated (IP) with antibodies recognizing Tubb3 (a) or Citron proteins (b). Immunoprecipitates and total lysates (Input) were then fractionated by SDS-PAGE and analyzed for western blotting (WB) with the indicated antibodies. (c) Total cell lysates from P4 WTand Cit-K KO mouse cerebellum were separated by 2D gel electrophoresis and analyzed by WB using the anti-Tubb3 TuJ antibody. The arrowhead indicates an acidic spot detected in the WT but not in the KO samples. The relative amount of protein in the acidic spot (phosphorylated) or in the main spot (nonphosphorylated) was quantified in the lower panel. (d) Total cell lysates from P4 WT mouse cerebellum, untreated (Ctrl) or treated with phage protein phosphatase- $\lambda$ (PP $\lambda$ ) or with erythrocyte protein phosphatase-2A (PP2A) were analyzed by 2D WB using the anti-Tubb3 antibodies TuJ or no-P444 (not recognizing the phosphorylated-S444 isoform). (e) Total cell lysates from HeLa cells transiently transfected with the indicated eGFP-TUBB3 fusion constructs were separated by 2D gel electrophoresis and analyzed by WB using the anti-GFP antibodies. The arrowhead indicates an acidic spot detected with the WT construct but not with the S444A mutant. (f and $\mathbf{g}$ ) HeLa cells were transiently transfected with eGFP or with the indicated eGFP fusion constructs and after $24 \mathrm{~h}$ were transfected with control (CTRL) or with CIT-K-specific siRNAs. After $48 \mathrm{~h}$, the percentage of binucleated cells was quantified as above. All the results are representative of at least three independent experiments ( 30 fields for each experiment). Error bars $=$ S.E.M. Statistical significance of differences in average was established using Student's two-tailed $t$-test. ${ }^{* *} P<0.01 ;{ }^{* * *} P<0.001$

pattern similar to the endogenous TUBB3 with a well visible acidic spot, whereas the latter is not detectable with the S444A mutant (Figure 5e). These results indicate that CIT-K promotes the phosphorylation of TUBB3 in proliferating neural tissue and in HeLa cells and are consistent with S444 being a major phosphorylation site influenced by CIT-K levels. We then evaluated the functional significance S444 phosphorylation for cytokinesis by expressing wild-type and 
mutant GFP fusions in HeLa cells and by measuring the number of transfected binucleated cells. Importantly, expression of the S444A mutant was sufficient to induce a significant increase of binucleated cells under control conditions (Figure 5f) and worsened the CIT-K knockdown phenotype (Figure $5 \mathrm{~g}$ ). Conversely, expression of a phospho-mimetic S444D mutant did not increase the number of binucleated cells in controls (Figure 5f) and rescued, rather than worsened, the CIT-K knockdown phenotype (Figure $5 \mathrm{~g}$ ). Wild-type and mutant proteins were expressed at levels comparable to the levels of endogenous TUBB3 (Supplementary Figure S2B) and displayed a similar localization pattern (not shown). As in the case of CIT-K depletion, time-lapse analysis of transfected cells revealed that the binucleation phenotype is produced by abscission failure (Supplementary Figure S4 and Supplementary Movies S3 and S4). These results strongly suggest that phosphorylation of $\mathrm{S} 444$ is a functionally relevant downstream event of the CIT-K pathway.

CK2 $a$ interacts with CIT-K, localizes at the midbody through CIT-K and affects cytokinesis through TUBB3 S444 phosphorylation. We next asked whether TUBB3 could be a direct target of CIT-K by performing in vitro kinase assays with constitutively active CIT-K isolated from insects cells and bacterially expressed TUBB3. In no case we detected increased activity over background (Supplementary Figure S5), whereas, as expected, ${ }^{46}$ the same kinase efficiently phosphorylated recombinant myosin light chain (Supplementary Figure S5). This result indicates that a CIT-Kassociated kinase should mediate CIT-K-dependent TUBB3 S444 phosphorylation. We therefore tested whether the two kinases that are known to phosphorylate this residue, that is, $\mathrm{CK} 2 a^{43}$ and G protein-coupled receptor kinase 2 (GRK2), ${ }^{47}$ are capable of associating with CIT-K. Interestingly, although no association could be detected with GRK2 (data not shown), endogenous CK2a was co-precipitated with overexpressed CIT-K or with its amino-terminal half, whereas it was not co-precipitated with the C-terminal half of CIT-K (Figure 6a). More importantly, endogenous CK2a was co-precipitated with endogenous CIT-K from both HeLa cells synchronized in mitosis (Figure 6b) and from P4 wild-type mouse cerebellum (Figure 6c). In addition, consistent with a recent report, ${ }^{48}$ we found that $\mathrm{CK} 2 a$ is enriched at the midbody of HeLa cells, where it colocalizes with CIT-K (Figure 6d). We therefore asked whether CIT-K is necessary for this localization. Strikingly, although CK2a was easily detectable in the central region of the midbody in $\sim 80 \%$ of late telophases HeLa cells transfected with control siRNAs, it was detected in $<20 \%$ of CIT-K-depleted cells (Figure $6 e$ ). Moreover, we found that the inactivation of CK2 $a$ by RNAi increases the number of binucleated cells (Figure 6f), suggesting that it may affect cytokinesis besides other described mitotic processes. ${ }^{49}$ Finally, we found that the increase of binucleated cells elicited by CK2a depletion is reverted almost to control levels by expressing the TUBB3 mutant S444D (Figure 6f). Altogether, these results are consistent with CK2 $a$ being the effector kinase of CIT-K in controlling TUBB3 S444 phosphorylation at the midbody.

\section{Discussion}

The differentiation program of many cell types is characterized by dramatic changes of the cytoskeleton that may potentially hinder the highly organized molecular machinery responsible for cytokinesis. A striking example of this possibility is the progressive accumulation of myofibrils and intermediate filaments in proliferating cardiomyocytes, ending with abortive cytokinesis and accumulation of binucleated cells during the early postnatal period of heart development. ${ }^{50,51}$

Therefore, it is conceivable that specific mechanisms may have evolved to cope with the modifications of cytoskeleton that could inhibit cytokinesis in some differentiating cell types. The case of CIT-K is a so far a unique example of how cytokinesis of specific cells can be affected by the lack of a particular protein. The results of previous studies ${ }^{21,26}$ and the present report indicate that neurogenic divisions are strongly dependent on CIT-K. The neuronal depletion of the most superficial cortical layers, ${ }^{21,26}$ the high levels of apoptosis in the IZ (Figure 1) and the frequent positivity of apoptotic cells for neuronal markers (Figure 1g) indicate that the most affected mitoses are symmetric neurogenic divisions. However, the high number of apoptotic cells in the ventricular and subventricular regions of developing neocortex and the Tbr2 and Pax6 positivity of some apoptotic cells suggest that the symmetric and asymmetric divisions of progenitors can also be affected. In this regard, it should be considered that the percentage of progenitor cells undergoing apoptosis could be significantly underestimated, because a high percentage of TUNEL-positive cells are negative to all markers and because the transcription factors Pax6 and Tbr2 could disappear from apoptotic cells more easily than the cytoskeletal marker TuJ. However, the huge difference in the frequency of apoptotic neurons and apoptotic progenitors (Figure 1g) indicates that dividing neuronal precursors become progressively more sensitive to CIT-K loss as they commit to terminal differentiation. A second conclusion of our study is that midbody microtubules are a primary target of CIT-K in abscission control. Although the involvement of CIT-K in events related to microtubule dynamics has previously been suggested, ${ }^{25,52}$ we provide for the first time evidence of a direct functional role. Indeed, we showed that CIT-K loss alters midbody microtubule stability in sensitive cells, whereas the stability of microtubules affects the sensitivity of cytokinesis to CIT-K loss (Figure 2). In addition to the previous studies implicating CIT-K in actindependent midbody stabilization, ${ }^{23,25,53}$ these results add strong support the notion that CIT-K plays a crucial role in the crosstalk between actin and microtubules responsible for the maturation of midbody that precedes abscission. ${ }^{23-25,54}$ The activity of CIT-K on microtubule stability is most likely independent from the reported interaction between CIT-K and KIF14. Indeed, although KIF14 may be functionally implicated in crosslinking midbody microtubules via its interaction with PRC1, ${ }^{25}$ it does not seem to affect their stability (Figures 2a and $b$ ). This result is not in contrast with the available data for three main reasons. The first is that the functional link between CIT-K and KIF14 seems stronger in Drosophila cells than in mammalian cells. ${ }^{25}$ The second is that even in Drosophila cells the cytokinesis phenotype produced by KIF14 depletion is significantly less severe than the CIT-K 
a

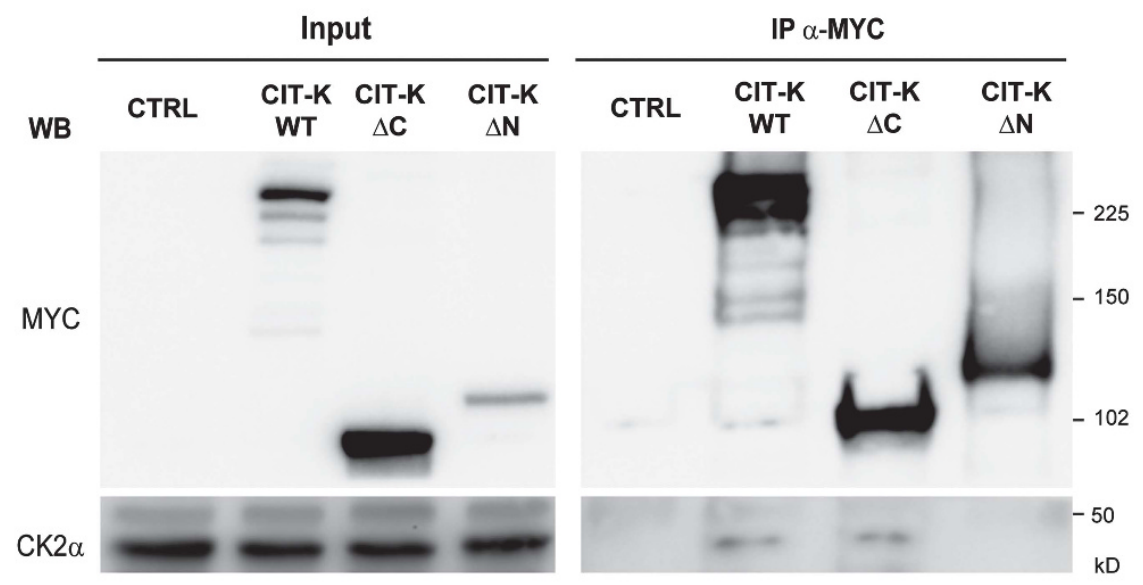

b
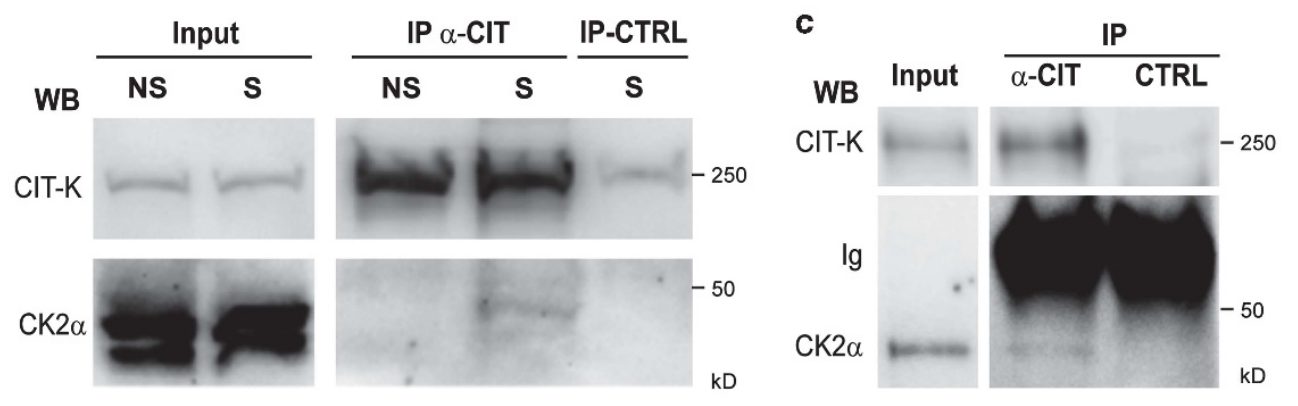

d

CIT-K CK $2 \alpha$ DAPI

CIT-K
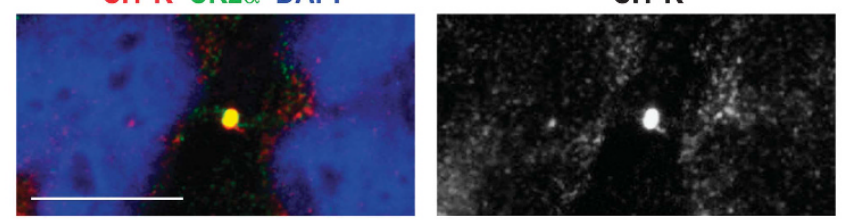

CK2 $\alpha$

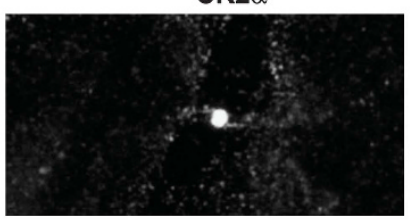

e

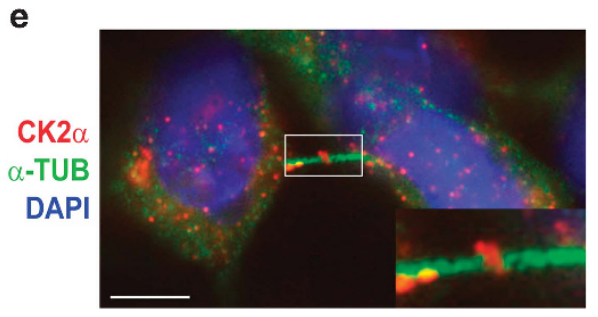

siRNA-Ctrl

f

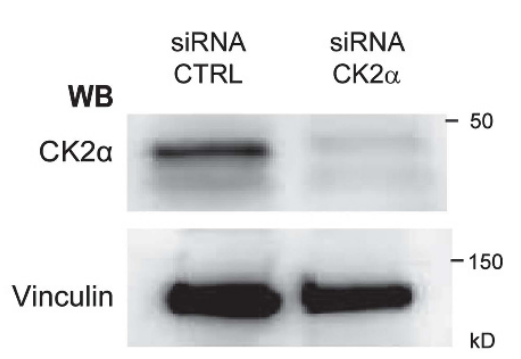

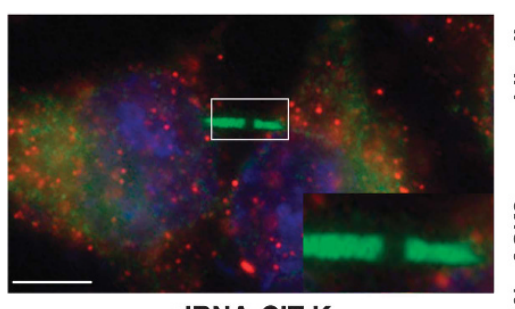

siRNA-CIT-K
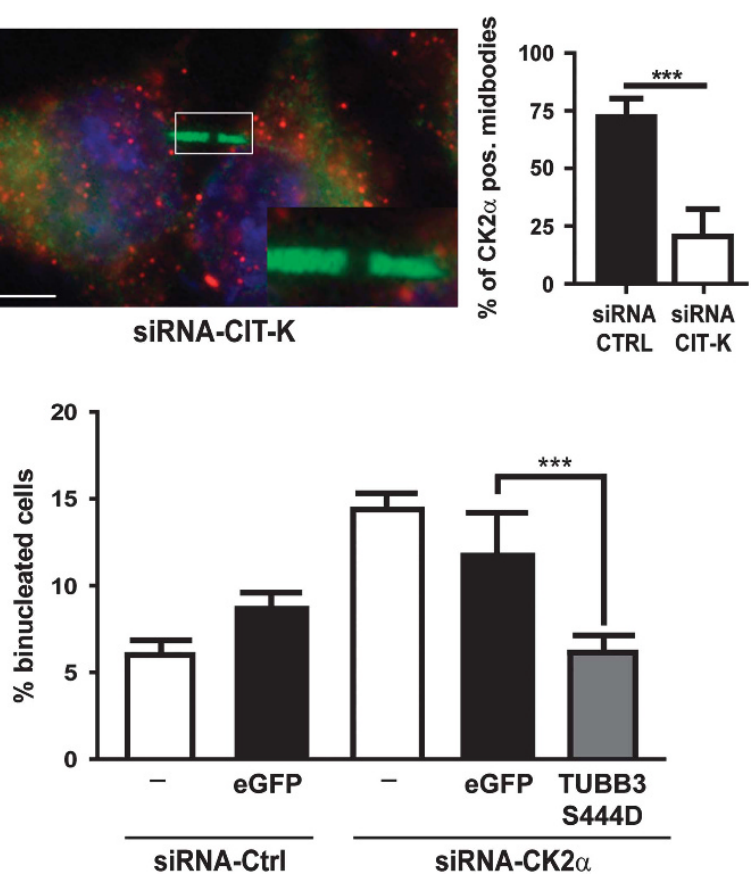
depletion phenotype, ${ }^{25}$ indicating that CIT-K plays additional roles in preparing the midbody for abscission.

Third, and perhaps most important conclusion of our study, is that the expression of TUBB3 in proliferating cells is a major determinant of the sensitivity to CIT-K loss. This finding is important because it may reconcile the apparent conflict between previous studies, concerning the general or tissuespecific role of CIT-K in cytokinesis of human cells. The phenotype of CIT-K loss in humans has not been reported yet, but as CIT-K knockdown leads to cytokinesis failure in HeLa cells, it was argued that tissue-specific cytokinesis failure is a rodent-specific feature, whereas in human cells CIT-K must play a general role. ${ }^{15}$ However, the finding that the sensitivity of HeLa cells to CIT-K loss can be strongly modulated by TUBB3 suggests an alternative possibility. Indeed, under physiological conditions, the expression of TUBB3 has only been reported in the CNS and testis, ${ }^{44,55}$ the only two organs highly sensitive to CIT-K inactivation. ${ }^{22}$ On the other hand, high expression levels of TUBB3 have been reported in many tumors and tumor cell lines, including HeLa cells. ${ }^{56,57}$ Therefore, we propose that the sensitivity to CIT-K loss is more dependent on TUBB3 expression than on speciesspecific molecular events. Whether the TUBB3-dependent effect of CIT-K on microtubule stability is localized to the midbody or is also spread to the mitotic cytoplasm could depend on cell-specific determinants, such as the levels of TUBB3 or other undefined factors. Indeed, the destabilization of microtubules induced by CIT-K loss is midbody specific in HeLa cells, whereas it also extends to cytoplasm in neuronal progenitors that may express TUBB3 levels higher than HeLa cells (Supplementary Figure $\mathrm{S} 1$ ). The latter finding raised the possibility that CIT-K may also affect other aspects of the mitotic process in neuronal progenitors, a hypothesis that will be interesting to test.

On this basis, we would predict that the loss of CIT-K in humans should result in a syndrome similar to the one observed in mice and rats, and that tumors expressing TUBB3 could be specifically sensitive to CIT-K inactivation. In addition, our data indicate that expression of TUBB3 in proliferating neuronal precursors, at levels much lower than the levels usually measured in young postmitotic neurons, could be functionally relevant. It will be very interesting to address whether the precocious TUBB3 expression is only a potentially detrimental phenomenon that may destabilize the midbody during cell divisions preceding neurogenesis or whether it may play a direct functional role during these mitoses. Mutations in TUBB3 have been implicated in developmental disorders of the CNS characterized by defective axon outgrowth or neuronal migration. ${ }^{38,58}$ On the basis of our data, it seems reasonable to speculate that mutations of the S444 could also affect neuronal progenitor proliferation, with consequences similar to those provoked by CIT-K loss.

How does CIT-K modulate midbody microtubule stability through TUBB3? On the basis of the data presented in Figures 5 and 6 , we propose that, by interacting with both TUBB3 and CK2a, CIT-K may bring in close proximity the two proteins, thus facilitating TUBB3 phosphorylation on S444. In HeLa cells the formation of this complex could largely be restricted to the midbody, thus explaining why the levels of phosphorylated TUBB3 are so low but still functionally significant. On the other hand, in neuronal progenitors, a higher concentration of TUBB3 could facilitate the interaction, making it more widespread and strongly increasing the ratio between phosphorylated and nonphosphorylated forms. However, even though it is currently believed that CK2 is not strongly regulated in its kinase activity, we cannot exclude that CIT-K may also modulate it. As CK2a is known to regulate many substrates crucial for cell proliferation and viability, it will be very interesting to study whether CIT-K may affect their phosphorylation state, locally or globally, and whether this mechanism may help to explain why cells that fail cytokinesis for CIT-K absence are lost by programmed cell death.

\section{Materials and Methods}

Cell culture and synchronization. HeLa cells were cultured in RPMI supplemented with $10 \%$ fetal bovine serum and penicillin/streptomycin at $37^{\circ} \mathrm{C}$ and in $5 \% \mathrm{CO}_{2}$. For synchronization, asynchronous cultures were supplemented with $2 \mathrm{mM}$ thymidine (Sigma, St. Louis, MO, USA) and maintained under these conditions for $16 \mathrm{hh}$ and then released for $4-6 \mathrm{~h}$ in fresh complete medium supplemented with $30 \mu \mathrm{M}$ deoxycytidine. After release, cells were cultured for a further $16 \mathrm{~h}$ in fresh complete medium in the presence of $50 \mathrm{ng} / \mathrm{ml}$ nocodazole (Sigma) to block cells at prometaphase. Finally, cells were washed twice with fresh medium and allowed to progress through mitosis/cytokinesis for the indicated times.

Neuronal progenitor cultures. Embryonic brains were isolated from E12 timed pregnant mice. The lateral portion of the dorsal telencephalon was dissected and dissociated using Papain and plated at the concentration of $5 \times 10^{4} \mathrm{cell} / \mathrm{s} / \mathrm{cm}^{2}$ on glass coverslip treated with Poly-L-lysine and Laminin. Cells were analyzed $18 \mathrm{~h}$ after plating. Culture media were composed of DMEM F12, supplemented with $2 \%$ B27 w/o retinoic acid, $10 \mathrm{ng} / \mathrm{ml}$ EGF and $40 \mathrm{ng} / \mathrm{ml}$ bFGF (all components were from Life Technologies, Carlsbad, CA, USA).

Plasmids, recombinant proteins, siRNAs and cell transfection. The Myc-CIT-K wild-type construct has been previously described. ${ }^{23}$ The mutant constructs were generated from full-length Myc-CIT-K and corresponded to amino acids 0-955 $(\Delta \mathrm{C})$ and 955-2055 $(\Delta \mathrm{N})$. The EGFP-TUBB3 expression vector was constructed by cloning the amplified coding sequence of human TUBB3 into the pEGFP-C1 (Takara-Clontech, Kyoto, Japan). Mutant TUBB3 constructs were obtained from the EGFP-TUBB3 plasmid by mutagenizing the S444 codon through the QuikChange Lightning Multi Site-Directed Mutagenesis Kit

Figure 6 Physical and functional interaction between CIT-K and CK2 $\alpha$ upstream of TUBB3 S444 phosphorylation. (a) HeLa cells were transiently transfected with MYC-tagged CIT-K wild-type or mutant constructs. Overexpressed proteins were immunoprecipitated using anti-MYC antibodies and analyzed by WB as indicated. (b) Total cell lysates from HeLa cells non syncronized (NS) or synchronized in mitosis by double thymidine block and nocodazole (S) were immunoprecipitated with control (CTRL) or antiCitron antibodies $(\alpha$-CIT). Immunoprecipitations (IP) and total lysates (Input) were then analyzed by WB with the indicated antibodies. (c) Total cell lysates from wild-type P4 mouse cerebellum $(2 \mathrm{mg}$ ) were immunoprecipitated as in (b). IP and total lysates (Input) were then analyzed by WB with the indicated antibodies. Ig, immunoglobulins. (d) Asynchronous HeLa cells were processed for immunofluorescence using the anti-CK2 $\alpha$ and anti-Citron antibodies. The two proteins colocalize at the midbody of late telophase cells. Scale bar $=5 \mu \mathrm{m}$. (e) Asynchronous HeLa cells were processed for immunofluorescence $48 \mathrm{~h}$ after transfection with the indicated siRNAs using the anti-CK2 $\alpha$ and anti- $\alpha$ tubulin $(\alpha$-TUB). The region containing the midbody of two dividing cells was magnified in the inset. Scale bar $=5 \mu \mathrm{m}$. (f) HeLa cells either nontransfected $(-)$ or transiently transfected with eGFP or with the S444D TUBB3 mutant were transfected after $24 \mathrm{~h}$ with control (Ctrl) or with CK2 $\alpha$-specific siRNAs. After $48 \mathrm{~h}$, the percentage of binucleated cells was quantified as described above. The knockdown of CK2 $\alpha$ was verified by WB (left panel). Scale bars $=10 \mu \mathrm{m} .{ }^{* * *} P<0.001$ 
(Stratagene, La Jolla, CA, USA). The pEGFP-EB1 expression vector (Tirnauer Lab, Farmington, CT, USA) was obtained from AddGene (Cambridge, MA, USA).

Knockdown of CIT-K was achieved using previously validated siRNA sequences ${ }^{15,23}$ obtained from GE Healthcare, Dharmacon (Lafayette, CO, USA). The knockdown of TUBB3, TUBB2A and KIF14 was achieved using the corresponding ON-TARGETplus SMART pools (GE Healthcare, Dharmacon). The ON-TARGETplus non-targeting siRNA \#1 (GE Healthcare, Dharmacon) was used as a negative control. HeLa cells plated on six-well plates were transfected using $1 \mu \mathrm{g}$ of the required plasmid DNA and $3 \mu \mathrm{l}$ TransIT-LT1 Transfection reagent (Mirus, Madison, WI, USA) or using $6.25 \mu \mathrm{l}$ of the required siRNA $(20 \mu \mathrm{M})$ and $10 \mu$ L Lipofectamine 2000 (Life Technologies) according to the manufacturers' specifications.

For transfection of mouse neuronal progenitors, $5 \times 10^{5}$ cells were nucleofected with Amaxa Rat Nucleofection Kit (VVPG-1003, Amaxa Biosystems, Cologne, Germany) using the program A033. siRNAs were used at the concentration of $200 \mathrm{nM}$.

Antibodies. The following antibodies were used: mouse monoclonal anti-Citron (Transduction Laboratories, BD Biosciences, San Jose, CA, USA) used for western blotting and immunofluorescence; rabbit polyclonal anti-Citron (Abcam, Cambridge, MA, USA) for immunoprecipitation; mouse monoclonal anti-Vinculin (Cell Signaling Technology, Danvers, MA, USA); mouse monoclonal and rabbit polyclonal antiTUBB3 TuJ1 (Covance, Princeton, NJ, USA); rabbit polyclonal anti-TUBB3 not reactive with the phosphorylated-S444 moiety (Abcam, cat. no. ab76288); rabbit polyclonal anti-Tbr2 (Abcam); mouse monoclonal anti-Pax6 (Santa Cruz Biotechnology, Dallas, TX, USA); mouse monoclonal anti-Nestin (Abcam); rat monoclonal anti-tyrosinated $\alpha$-tubulin YL1/2 (Abcam); mouse monoclonal antiacetylated $\alpha$-tubulin 6-11B-1 (Abcam); rabbit polyclonal anti-TUBB2A (Sigma); mouse monoclonal anti-Aurora B (Transduction Laboratories, BD Biosciences); mouse monoclonal anti-MYC clone 9E10 (Santa Cruz Biotechnology); rabbit polyclonal anti-CK2 $\alpha$ (Sigma); rabbit polyclonal anti-CK2 $\alpha$ (Abcam, cat. no. ab70774); and rabbit polyclonal anti-KIF14 (Abcam, cat. no. ab3746).

Immunofluorescence on cell cultures. Cells were fixed in methanol at $-20^{\circ} \mathrm{C}$ for $2 \mathrm{~min}$. Cells were then permeabilized with $0.1 \%$ (vol/vol) Triton X-100/ PBS for $10 \mathrm{~min}$, blocked for $30 \mathrm{~min}$ at RT in blocking solution (PBS, 5\% BSA) and incubated for $1 \mathrm{~h}$ with the primary antibodies. Primary antibodies were detected with anti-rabbit Alexa 488 or 568, anti-mouse Alexa 488 or anti-rat 568 (Invitrogen, Waltham, MA, USA) used in a 1:1000 dilution. To visualize DNA, fixed cells were stained with DAPI (Sigma). Images were acquired using an ApoTome system (Zeiss, Oberkochen, Germany) or with an SP5 confocal microscope (Leica, Oberkochen, Germany).

Immunofluorescence of neocortical brain slices. Brains from E14.5 embryos were dissected and washed in PBS and then fixed overnight at $4^{\circ} \mathrm{C}$ in $4 \%$ paraformaldehyde, $\mathrm{pH} 7.2$. Brains were then equilibrated for $12-24 \mathrm{~h}$ at $4^{\circ} \mathrm{C}$ in $30 \%$ sucrose in PBS, embedded in OCT compound (VWR, Radnor, PA, USA) and stored at $-20^{\circ} \mathrm{C}$. Cryostat sections $(20 \mu \mathrm{m})$ were rehydrated with PBS and incubated for $1 \mathrm{~h}$ at $70^{\circ} \mathrm{C}$ in antigen retrieval solution (sodium citrate $10 \mathrm{mM}, \mathrm{pH}$ 6.0). Sections were permeabilized with $0.3 \%$ Triton $X-100$ in PBS for $30 \mathrm{~min}$, quenched with $0.1 \mathrm{M}$ glycine-Tris $\mathrm{pH} 7.4$ for $30 \mathrm{~min}$ and incubated with blocking solution $(0.3 \%$ Triton $\mathrm{X}-100$ and $0.2 \%$ gelatin in a $3: 7$ mixture of $1 \mathrm{M} \mathrm{NaCl}$ and PBS) for $30 \mathrm{~min}$ followed by overnight incubation with primary antibodies at $4{ }^{\circ} \mathrm{C}$, then washed and incubated for $30 \mathrm{~min}$ at room temperature with the corresponding secondary antibody (Life Technologies) and DAPI to counterstain nuclei and finally mounted using Prolong antifade reagent (Life Technologies). To analyze the presence of Tubb3 in the midbody of neuronal progenitors in embryonal slice, midbodies were selected using the freehand tool of Fiji software ${ }^{59}$ on AIM1 staining (midbody marker). Colocalization analysis was then performed using Fiii plug in COLOC 2, and Manders value of colocalization was obtained for AIM1 and TuJ.

Immunoprecipitations and western blotting. For all immunoprecipitations, cells and tissue were extracted with lysis buffer containing $1 \%$ Triton X-100, $120 \mathrm{mM}$ sodium chloride, $50 \mathrm{mM}$ Tris- $\mathrm{HCl}$ (pH 7.5), protease inhibitors (Roche, Basel, Switzerland) and $1 \mathrm{mM}$ phenylmethylsulfonyl fluoride (PMSF). The extracts were then clarified by centrifugation. Antibodies and protein-A or G-Sepharose beads (GE Healthcare Life Science, Little Chalfont, UK) were added to lysates and incubated overnight at $4^{\circ} \mathrm{C}$. Pellets were washed four times with lysis buffer and analyzed by SDS-PAGE. For immunoblots, immunoprecipitates or equal amounts of proteins from total cell lysates were resolved by reducing SDS-PAGE and transferred to nitrocellulose filters that were then incubated with the indicated antibodies and developed using the ECL system (Merck Millipore, Billerica, MA, USA).

Live-imaging experiments. S3 HeLa cells stably expressing RFP- $\alpha$-tubulin were a kind gift of Ivan Stamenkovic (University of Lausanne, Lausanne, Switzerland). FRAP experiments were performed on midbodies of constant size using $100 \%$ transmission of $568 \mathrm{~nm}$ laser for photobleaching. Recovery kinetics were then measured and, after background subtraction, were normalized to pre- and last post-bleach frames. ${ }^{30}$ HeLa cells were transfected with pEGFP-EB1 expression construct or with GFP-TUBB3 fusion constructs. Time-lapse imaging of EB1 comets was performed using $63 \times 1.3$ objective at 1 frame $/ 500 \mathrm{~ms}$ for $2 \mathrm{~min}$ on an SP5 confocal microscope (Leica). Tracking and speed analysis was done with ImageJ tracking tools. Time-lapse imaging of cell division was performed under an Axiovert Zeiss inverted microscope equipped with an environmental chamber using a $20 \times$ objective. Cultures were maintained at $37^{\circ} \mathrm{C}$ and gassed with $5 \% \mathrm{CO}_{2}$. For phase contrast imaging, exposure times were typically $5 \mathrm{~ms}, 20$ frames per $h$.

2D western blotting and phosphatase assays. Protein separation in the first dimension was performed on a Protean IEF cell (Bio-Rad, Hercules, CA, USA) using IPG strips of pH 3-10NL (Ready Strip; $0.5 \times 3 \times 170 \mathrm{~mm}$, Bio-Rad). Then, $2 \mathrm{mg}$ proteins from mouse cerebella or HeLa cells were dissolved in $300 \mu \mathrm{l}$ of 2D sample buffer (7 M urea, $2 \mathrm{M}$ thiourea, 4\% (w/v) CHAPS, 1.6\% (w/v) DTT, 2\% (v/ v) biolytes, and $1.2 \%$ (v/v) Bio-Rad reagent). The IPG strips were rehydrated at $50 \mathrm{~V}$ for $12 \mathrm{~h}$, followed by focusing for a total of $100 \mathrm{kVh}$ at $18^{\circ} \mathrm{C}$. The IPG strips were then transferred on to the top of a $10 \%$ polyacrylamide gel (acrylamide/ bisacrylamide $37.5: 1,2.6 \%$ crosslinker; gel dimension, $8 \mathrm{~cm} \times 6 \mathrm{~cm} \times 1.5 \mathrm{~mm}$ ). Electrophoresis in the second dimension was performed in a Protean II xi system (Bio-Rad) using $1 \times$ Tris/Glycine/SDS buffer at $100 \mathrm{~V}$ at $15^{\circ} \mathrm{C}$ until the bromophenol blue dye front was on the bottom of the gel.

Treatments with erythrocyte PP2A (Merck Millipore) and $\lambda$-phosphatase (New England Biolabs, Ipswich, MA, USA) were performed in dephosphorylation buffer that contained $0.1 \mathrm{M}$ MES, $0.1 \mathrm{mM}$ EDTA, $1 \mathrm{mM}$ EGTA, $0.5 \mathrm{mM} \mathrm{MgCl} 2$ and $1 \mathrm{mM} \beta$ mercaptoethanol, $\mathrm{pH} 6.4$. In the case of $\lambda$-phosphatase, the buffer also contained $2 \mathrm{mM} \mathrm{MnCl}$. Then, $1.6 \mathrm{IU}$ of PP2A or 400 units of $\lambda$-phosphatase were incubated with cerebellum lysates $\left(2 \mathrm{mg}\right.$ of proteins) at $30^{\circ} \mathrm{C}$ for $20 \mathrm{~min}$, followed by the addition of another aliquot of the phosphates and incubation for another $30 \mathrm{~min}$. The PP2A dephosphorylation was terminated with $5 \mu \mathrm{M}$ calyculin A. Completion of dephosphorylation by $\lambda$-phosphatase was confirmed by performing an anti-phosphotyrosine blot on $30 \mu \mathrm{g}$ of the reaction.

In vitro kinase assay. Recombinant GST-MLC was obtained from bacteria expressing the corresponding expression vector (kind gift of Dr. Kozo Kaibuchi, Nagoya, Japan). Recombinant GST-CIT-SK was obtained using the BD-Pharmingen (San Jose, CA, USA) baculovirus expression system by subcloning the mouse Cit-SK coding sequence (GeneBank accession no. AF086823) in the pAcG2T Transfer Vector. In both cases, GST fusion proteins were isolated from total cell lysates of bacterial and insect cells through Glutathione Sepharose 4B (GE Healthcare Lifesciences) affinity chromatography according to the manufacturer's specifications. Recombinant bacterial MBP-TUBB3 expression plasmid was obtained by subcloning the TUBB3 coding sequence into the pMAL plasmid (New England Biolabs, USA) and by performing amylose affinity chromatography according to the manufacturer's specifications. For in vitro kinase assay, recombinant proteins were incubated in $50 \mu \mathrm{l}$ of kinase buffer (50 mm HEPES, pH 7.4, $5 \mathrm{mmMgCl}_{2}, 3 \mathrm{~mm} \mathrm{MnCl}$, plus $0.1 \mathrm{~mm} \mathrm{ATP}$ and $10 \mathrm{mCi}$ of [ $\gamma$-32P]ATP $\left(6000 \mathrm{Ci} / \mathrm{mm}\right.$, Amersham) for $30 \mathrm{~min}$ at $30^{\circ} \mathrm{C}$. The products were analyzed by 5 or $12.5 \%$ SDS-PAGE followed by autoradiography.

\section{Conflict of Interest}

The authors declare no conflict of interest.

Acknowledgements. This work was supported by the Telethon Foundation through grant no. GGP12095 to FDC.

1. Barr FA, Gruneberg U. Cytokinesis: placing and making the final cut. Cell 2007; 131: 847-860.

2. Glotzer M. The molecular requirements for cytokinesis. Science 2005; 307: 1735-1739.

3. Schiel JA, Prekeris R. Membrane dynamics during cytokinesis. Curr Opin Cell Biol 2013; 25: 92-98. 
4. Fededa J P, Gerlich DW. Molecular control of animal cell cytokinesis. Nat Cell Biol 2012; 14: 440-447.

5. Balasubramanian MK, Srinivasan R, Huang Y, Ng KH. Comparing contractile apparatus-driven cytokinesis mechanisms across kingdoms. Cytoskeleton (Hoboken) 2012; 69: 942-956.

6. Lacroix B, Maddox AS. Cytokinesis, ploidy and aneuploidy. J Pathol 2012; 226: 338-351.

7. Griswold MD, Oatley JM. Concise review: defining characteristics of mammalian spermatogenic stem cells. Stem Cells 2013; 31: 8-11.

8. Knust E, Huttner WB. Cell polarity from cell division. Dev Cell 2007; 12: 664-666.

9. Kosodo Y, Toida K, Dubreuil V, Alexandre P, Schenk J, Kiyokage E et al. Cytokinesis of neuroepithelial cells can divide their basal process before anaphase. EMBO J 2008; 27: 3151-3163.

10. Jordan SN, Canman JC. Rho GTPases in animal cell cytokinesis: an occupation by the one percent. Cytoskeleton (Hoboken) 2012; 69: 919-930.

11. Piekny A, Werner M, Glotzer M. Cytokinesis: welcome to the Rho zone. Trends Cell Biol 2005; 15: 651-658.

12. Chircop M. Rho GTPases as regulators of mitosis and cytokinesis in mammalian cells. Small GTPases 2014; 5: 1-14.

13. Madaule P, Eda M, Watanabe N, Fujisawa K, Matsuoka T, Bito $\mathrm{H}$ et al. Role of citron kinase as a target of the small GTPase Rho in cytokinesis. Nature 1998; 394: 491-494.

14. Di Cunto F, Calautti E, Hsiao J, Ong L, Topley G, Turco E et al. Citron rho-interacting kinase, a novel tissue-specific ser/thr kinase encompassing the Rho-Rac-binding protein Citron. J Biol Chem 1998; 273: 29706-29711.

15. Gruneberg U, Neef R, Li X, Chan EH, Chalamalasetty RB, Nigg EA et al. KIF14 and citron kinase act together to promote efficient cytokinesis. J Cell Biol 2006; 172: 363-372.

16. Naim V, Imarisio S, Di Cunto F, Gatti M, Bonaccorsi S. Drosophila citron kinase is required for the final steps of cytokinesis. Mol Biol Cell 2004; 15: 5053-5063.

17. D'Avino PP, Savoian MS, Glover DM. Mutations in sticky lead to defective organization of the contractile ring during cytokinesis and are enhanced by Rho and suppressed by Rac. J Cell Biol 2004; 166: 61-71.

18. Shandala T, Gregory SL, Dalton HE, Smallhorn M, Saint R. Citron kinase is an essential effector of the Pbl-activated Rho signaling pathway in Drosophila melanogaster. Development 2004; 131: 5053-5063.

19. Echard A, Hickson GR, Foley E, O'Farrell PH. Terminal cytokinesis events uncovered after an RNAi screen. Curr Biol 2004; 14: 1685-1693.

20. Sarkisian MR, Li W, Di Cunto F, D'Mello SR, LoTurco JJ. Citron-kinase, a protein essential to cytokinesis in neuronal progenitors, is deleted in the flathead mutant rat. J Neurosci 2002; 22: RC217.

21. Di Cunto F, Imarisio S, Hirsch E, Broccoli V, Bulfone A, Migheli A et al. Defective neurogenesis in citron kinase knockout mice by altered cytokinesis and massive apoptosis. Neuron 2000; 28: 115-127.

22. Di Cunto F, Imarisio S, Camera P, Boitani C, Altruda F, Silengo L. Essential role of citron kinase in cytokinesis of spermatogenic precursors. J Cell Sci 2002; 115: 4819-4826.

23. Gai M, Camera P, Dema A, Bianchi F, Berto G, Scarpa E et al. Citron kinase controls abscission through RhoA and anillin. Mol Biol Cell 2011; 22: 3768-3778.

24. Bassi ZI, Verbrugghe KJ, Capalbo L, Gregory S, Montembault E, Glover DM et al. Sticky/ Citron kinase maintains proper RhoA localization at the cleavage site during cytokinesis. J Cell Biol 2011; 195: 595-603.

25. Bassi ZI, Audusseau M, Riparbelli MG, Callaini G, D'Avino PP. Citron kinase controls a molecular network required for midbody formation in cytokinesis. Proc Natl Acad Sci USA 2013; 110: 9782-9787.

26. LoTurco JJ, Sarkisian MR, Cosker L, Bai J. Citron kinase is a regulator of mitosis and neurogenic cytokinesis in the neocortical ventricular zone. Cereb Cortex 2003: 13:588-591.

27. Sessa A, Mao CA, Hadjantonakis AK, Klein WH, Broccoli V. Tbr2 directs conversion of radial glia into basal precursors and guides neuronal amplification by indirect neurogenesis in the developing neocortex. Neuron 2008; 60: 56-69.

28. Witte $H$, Neukirchen $D$, Bradke $F$. Microtubule stabilization specifies initial neuronal polarization. J Cell Biol 2008; 180: 619-632.

29. Westermann S, Weber K. Post-translational modifications regulate microtubule function. Nat Rev Mol Cell Biol 2003; 4: 938-947.

30. Carrero G, McDonald D, Crawford E, de Vries G, Hendzel MJ. Using FRAP and mathematical modeling to determine the in vivo kinetics of nuclear proteins. Methods 2003; 29: 14-28.

31. Stepanova T, Slemmer J, Hoogenraad CC, Lansbergen G, Dortland B, De Zeeuw Cl et al. Visualization of microtubule growth in cultured neurons via the use of EB3-GFP (end-binding protein 3-green fluorescent protein). J Neurosci 2003; 23: 2655-2664.

32. Yu W, Baas PW. The growth of the axon is not dependent upon net microtubule assembly at its distal tip. J Neurosci 1995; 15: 6827-6833.

33. Vasquez RJ, Howell B, Yvon AM, Wadsworth P, Cassimeris L. Nanomolar concentrations of nocodazole alter microtubule dynamic instability in vivo and in vitro. Mol Biol Cell 1997; 8: 973-985.
34. Aprea J, Prenninger S, Dori M, Ghosh T, Monasor LS, Wessendorf E et al. Transcriptome sequencing during mouse brain development identifies long non-coding RNAs functionally involved in neurogenic commitment. Embo J 2013; 32: 3145-3160.

35. Falconer MM, Echeverri CJ, Brown DL. Differential sorting of beta tubulin isotypes into colchicine-stable microtubules during neuronal and muscle differentiation of embryonal carcinoma cells. Cell Motil Cytoskeleton 1992; 21: 313-325.

36. Khan IA, Luduena RF. Phosphorylation of beta III-tubulin. Biochemistry 1996; 35 3704-3711.

37. Menezes JR, Luskin MB. Expression of neuron-specific tubulin defines a novel population in the proliferative layers of the developing telencephalon. J Neurosci 1994; 14: 5399-5416.

38. Saillour Y, Broix L, Bruel-Jungerman E, Lebrun N, Muraca G, Rucci J et al. Beta tubulin isoforms are not interchangeable for rescuing impaired radial migration due to Tubb3 knockdown. Hum Mol Genet 2014; 23: 1516-1526.

39. Memberg SP, Hall AK. Dividing neuron precursors express neuron-specific tubulin. J Neurobiol 1995; 27: 26-43.

40. Calegari F, Haubensak W, Haffner C, Huttner WB. Selective lengthening of the cell cycle in the neurogenic subpopulation of neural progenitor cells during mouse brain development J Neurosci 2005; 25: 6533-6538.

41. Narvi E, Jaakkola K, Winsel S, Oetken-Lindholm C, Halonen P, Kallio L et al. Altered TUBB3 expression contributes to the epothilone response of mitotic cells. Br J Cancer 2013; 108: $82-90$.

42. Camera P, da Silva JS, Griffiths G, Giuffrida MG, Ferrara L, Schubert V et al. Citron-N is a neuronal Rho-associated protein involved in Golgi organization through actin cytoskeleton regulation. Nat Cell Biol 2003; 5: 1071-1078.

43. Diaz-Nido J, Serrano L, Lopez-Otin C, Vandekerckhove J, Avila J. Phosphorylation of a neuronal-specific beta-tubulin isotype. J Biol Chem 1990; 265: 13949-13954.

44. Lee MK, Rebhun LI, Frankfurter A. Posttranslational modification of class III beta-tubulin Proc Natl Acad Sci USA 1990; 87: 7195-7199.

45. Fanarraga ML, Avila J, Zabala JC. Expression of unphosphorylated class III beta-tubulin isotype in neuroepithelial cells demonstrates neuroblast commitment and differentiation. Eur J Neurosci 1999; 11: 517-527.

46. Yamashiro S, Totsukawa G, Yamakita Y, Sasaki Y, Madaule P, Ishizaki T et al. Citron kinase, a Rho-dependent kinase, induces di-phosphorylation of regulatory light chain of myosin II. Mol Biol Cell 2003; 14: 1745-1756.

47. Yoshida N, Haga K, Haga T. Identification of sites of phosphorylation by G-protein-coupled receptor kinase 2 in beta-tubulin. Eur J Biochem 2003; 270: 1154-1163.

48. Salvi M, Raiborg C, Hanson PI, Campsteijn C, Stenmark H, Pinna LA. CK2 involvement in ESCRT-III complex phosphorylation. Arch Biochem Biophys 2014; 545: 83-91.

49. St-Denis NA, Derksen DR, Litchfield DW. Evidence for regulation of mitotic progression through temporal phosphorylation and dephosphorylation of CK2alpha. Mol Cell Biol 2009; 29: 2068-2081.

50. Rumyantsev PP. Electron microscope study of the myofibril partial disintegration and recovery in the mitotically dividing cardiac muscle cells. ZZellforsch Mikrosk Anat 1972; 129: 471-499.

51. Brodsky WY, Arefyeva AM, Uryvaeva IV. Mitotic polyploidization of mouse heart myocytes during the first postnatal week. Cell Tissue Res 1980; 210: 133-144.

52. Maliga Z, Junqueira M, Toyoda Y, Ettinger A, Mora-Bermúdez F, Klemm RW et al. A genomic toolkit to investigate kinesin and myosin motor function in cells. Nat Cell Biol 2013; 15 325-334.

53. Watanabe S, De Zan T, Ishizaki T, Narumiya S. Citron kinase mediates transition from constriction to abscission through its coiled-coil domain. J Cell Sci 2013; 126: 1773-1784.

54. D'Avino PP, Giansanti MG, Petronczki M. Cytokinesis in animal cells. Cold Spring Harb Perspect Biol 2015; 7: a015834.

55. Lewis SA, Cowan NJ. Complex regulation and functional versatility of mammalian alpha- and beta-tubulin isotypes during the differentiation of testis and muscle cells. J Cell Biol 1988; 106: 2023-2033.

56. Shibazaki M, Maesawa C, Akasaka K, Kasai S, Yasuhira S, Kanno K et al. Transcriptional and post-transcriptional regulation of betalll-tubulin protein expression in relation with cell cycle-dependent regulation of tumor cells. Int J Oncol 2012; 40: 695-702.

57. Newton CN, DeLuca JG, Himes RH, Miller HP, Jordan MA, Wilson L. Intrinsically slow dynamic instability of HeLa cell microtubules in vitro. J Biol Chem 2002; 277: 42456-42462.

58. Tischfield MA, Baris HN, Wu C, Rudolph G, Van Maldergem L, He W et al. Human TUBB3 mutations perturb microtubule dynamics, kinesin interactions, and axon guidance. Cell 2010; 140: 74-87.

59. Schindelin J, Arganda-Carreras I, Frise E, Kaynig V, Longair M, Pietzsch T et al. Fiji: an open-source platform for biological-image analysis. Nat Methods 2012; 9: 676-682.

Supplementary Information accompanies this paper on Cell Death and Differentiation website (http://www.nature.com/cdd) 